OPEN ACCESS

Edited by:

Basilios Tsikouras,

Universiti Brunei Darussalam, Brunei

Reviewed by:

Toshiaki Tsunogae,

University of Tsukuba, Japan

Gianluca Vignaroli,

University of Bologna, Italy

*Correspondence:

Alongkot Fanka

alongkot.f@chula.ac.th;

alongkotf@gmail.com

Specialty section:

This article was submitted to Structural Geology and Tectonics,

a section of the journal

Frontiers in Earth Science

Received: 20 April 2020

Accepted: 15 June 2020

Published: 14 July 2020

Citation:

Hunyek $V$, Sutthirat $C$ and Fanka A (2020) Magma Genesis and Arc Evolution at the Indochina Terrane

Subduction: Petrological and Geochemical Constraints From the Volcanic Rocks in Wang Nam Khiao Area, Nakhon Ratchasima, Thailand.

Front. Earth Sci. 8:271.

doi: 10.3389/feart.2020.00271

\section{Magma Genesis and Arc Evolution at the Indochina Terrane Subduction: Petrological and Geochemical Constraints From the Volcanic Rocks in Wang Nam Khiao Area, Nakhon Ratchasima, Thailand}

\author{
Vanachawan Hunyek, Chakkaphan Sutthirat and Alongkot Fanka* \\ Department of Geology, Faculty of Science, Chulalongkorn University, Bangkok, Thailand
}

Volcanic rocks and associated dikes have been exposed in Wang Nam Khiao area, Nakhon Ratchasima Province, northeastern Thailand where complex tectonic setting was reported. These volcanic rocks are classified as rhyolite, dacite, and andesite whereas dikes are also characterized by andesitic composition. These dikes clearly cut into the volcanic rocks and Late Permian hornblende granite in the adjacent area. Rhyolite and dacite are composed of abundant plagioclase and quartz whereas andesite and andesitic dike contain mainly plagioclase and hornblende with minor quartz. The volcanic rocks typically show plagioclase and hornblende phenocrysts embedded in fine-grained quartz and glass groundmass whereas dike rocks contain less glass matrix with more albitic laths. P-T conditions of crystallization are estimated, on the basis of Alin-hornblende geobarometry and hornblende geothermometry, at about 4.5-5.5 kbar, $861-927^{\circ} \mathrm{C}$ and $4.8-5.5 \mathrm{kbar}, 873-890^{\circ} \mathrm{C}$ for the magma intrusions that fed volcanic rocks and andesitic dikes, respectively. Whole-rock geochemistry indicates that these rock suites are related to calc-alkaline hydrous magma. The enriched large-ion lithophile elements (LILE; e.g., Rb, Sr) and depleted high-field-strength elements (HFSE; e.g., $\mathrm{Nb}, \mathrm{Ce}, \mathrm{Ti})$, with similar rare earth element (REE) patterns indicate arc magmatism. The results of this study are comparable with the continental arc magmatism along the Loei Fold Belt (LFB) as a consequence of the Late Permian Palaeo-Tethys subducted beneath Indochina Terrane.

Keywords: petrology, geochemistry, volcanic rock, Loei Fold Belt, Thailand

\section{INTRODUCTION}

Thailand and SE Asia are assembled from several tectonic terranes across from east to west (Bunopas, 1981; Charusiri et al., 2002; Sone and Metcalfe, 2008) including Indochina Terrane (Bunopas, 1981; Charusiri et al., 2002; Sone and Metcalfe, 2008), Loei Fold Belt (LFB; Bunopas, 1981), Sukhothai Terrane (Sone and Metcalfe, 2008), and Sibumasu (Sone and Metcalfe, 2008) or Shan-Thai Terrane (Bunopas, 1981; Charusiri et al., 2002), respectively (Figure 1). These terranes 
present diverse magmatic rocks, both plutonic (e.g., Cobbing et al., 1986, 1992; Nakapadungrat and Putthapiban, 1992; Charusiri et al., 1993; Searle et al., 2012; Ng et al., 2015a,b) and volcanic rocks (e.g., Jungyusuk and Khositanont, 1992; Intasopa, 1993; Intasopa and Dunn, 1994; Panjasawatwong et al., 2006; Barr and Charusiri, 2011; Boonsoong et al., 2011) corresponding to tectonic events in several episodes (Bunopas, 1981; Charusiri et al., 2002).

The LFB is located along the western edge of the Indochina Terrane (Figures 1, 2). In LFB, plutonic rocks have been defined as the Eastern Granite Belt of Thailand (Nakapadungrat and Putthapiban, 1992; Charusiri et al., 1993) whereas volcanic rocks, mostly reported in the northern part of LFB especially in Loei (Panjasawatwong et al., 2006; Kromkhun et al., 2013), Phetchabun (Boonsoong et al., 2011; Vivatpinyo et al., 2014), and Nakhon Sawan (Khositanont et al., 2008) areas, belong to Loei-Phetchabun-Ko Chang Volcanic Belt (Jungyusuk and Khositanont, 1992) (Figure 2). The main Late Permian-Triassic, Devonian-Carboniferous, and Silurian volcanic rocks have also been reported (Intasopa and Dunn, 1994; Panjasawatwong et al., 2006; Zaw et al., 2007; Boonsoong et al., 2011). These rocks resulted from amalgamation of tectonic terranes containing multiple generations of successive arc-related magmatic events (Charusiri et al., 2002; Zaw et al., 2014). Therefore, the magmatic rocks through the LFB, especially in the central and southern parts lacking of geological study (Figure 2), are very important to understand magmatism and tectonic evolution of the western Indochina Terrane.

The study area, Wang Nam Khiao in Nakhon Ratchasima province, Central Thailand, is located in the critical junction of several tectonic terranes (Figure 1A) where volcanic rocks and related dikes are also exposed. Detailed investigation of these rocks has never been reported; therefore, this study provides the results coming from petrology, mineral chemistry, and whole-rock geochemistry of these rocks before reconstruction of magmatic process and tectonic model of the area. The results are used to constrain the magmatic-tectonic scenario of central Thailand in the frame of the Indochina Terrane amalgamation during Carboniferous-Triassic.

\section{GEOLOGICAL SETTING}

Pre-Cenozoic volcanic, pyroclastic, and hypabyssal rocks in Thailand have been reported (e.g., Jungyusuk and Khositanont, 1992; Barr and Charusiri, 2011) mostly in the northern parts of Thailand (Figure 2). These rocks are divided into four volcanic belts (Jungyusuk and Khositanont, 1992) including Loei-Phetchabun-Ko Chang, Nan-Uttaradit, Chiang KhongTak, and Chiang Rai-Chiang Mai Volcanic Belts placing along $\mathrm{N}-\mathrm{S}$ from east to west, respectively (Figure 2).

Loei-Phetchabun-Ko Chang Volcanic Belt extends from Loei southward to Phetchabun, Nakhon Sawan, Wang Nam Khiao (the study area), and Ko Chang island in the Gulf of Thailand (Figure 2). Volcanic rocks in Loei area are divided into three subbelts including the eastern, central, and western sub-belts, based on their magmatic events (Intasopa and Dunn, 1994). The eastern volcanic sub-belt is characterized mainly by rhyolite occurred in a magmatic arc environment in Early Silurian (zircon U-Pb ages of $428 \pm 7,434 \pm 4$ Ma by Khositanont et al., 2008) or Late Devonian ( $\mathrm{Rb}-\mathrm{Sr}$ isochron age of $374 \pm 33 \mathrm{Ma}$ by Intasopa and Dunn, 1994). The central volcanic sub-belt contains mainly pillow basalts, hyaloclastite, and breccia, which have formed during Late Devonian ( $\mathrm{Rb}-\mathrm{Sr}$ isochron age of $361 \pm 11 \mathrm{Ma}$ by Intasopa and Dunn, 1994) as mid-oceanic ridge and island-arc basalts (Panjasawatwong et al., 2006). The western volcanic subbelt contains many types of rocks varying from andesite to dacite (Intasopa and Dunn, 1994; Boonsoong et al., 2011; Kromkhun et al., 2013), which extend to Phetchabun area (Boonsoong et al., 2011; Kamvong et al., 2014; Salam et al., 2014) and Laos (Qian et al., 2015). These volcanic rocks occurred as arc magmatism during Carboniferous to Middle Triassic (Zaw et al., 2007, 2014; Khositanont et al., 2008; Salam et al., 2014). Their ages were determined by zircon U-Pb dating such as of 230-254 Ma (Khositanont et al., 2008), 245.9-241.0 Ma (Kamvong et al., 2014), 245.9-258.6 Ma (Salam et al., 2014), 238-323 Ma (Zaw et al., 2007, 2014), and 314.6-349.6 Ma (Qian et al., 2015). In terms of the geochronology and geochemistry, this arc volcanism is comparable with the arc magmatism of plutonic rocks in Carboniferous to Triassic (Salam et al., 2014; Zaw et al., 2014; Fanka et al., 2016, 2018).

In the study area, Permo-Triassic volcanic rocks have also been reported (Putthaphiban et al., 1989a,b; Kemlheg and Wichidchalermpong, 1992a,b) in association with a plutonic complex (Fanka, 2016; Fanka et al., 2016, 2018). This complex includes hornblendite, hornblende gabbro, biotite granite, hornblende granite, and biotite-hornblende granite, defined as multiple arc magmatic events resulting from a PalaeoTethys subduction beneath Indochina Terrane during Late Carboniferous to Early Permian (zircon U-Pb ages of 314$285 \mathrm{Ma}$ from biotite granite), Late Permian (zircon $\mathrm{U}-\mathrm{Pb}$ ages of 253-257 Ma from hornblende granite and hornblende gabbro), and Middle Triassic (zircon $\mathrm{U}-\mathrm{Pb}$ ages of $237 \mathrm{Ma}$ from biotitehornblende granite) (Fanka et al., 2018).

\section{MATERIALS AND METHODS}

Representative volcanic rocks and related dikes were collected from the unweathered zones of Wang Nam Khiao area, Nakhon Ratchasima, northeast Thailand, for petrographic description, and mineral chemical and whole-rock geochemical analyses.

Microprobe analyses were performed on polished-thin sections using an electron probe micro-analyzer (EPMA JEOL JXA-8100). Analytical conditions were set up at $15 \mathrm{kV}$ and about $2 \mu \mathrm{A}$ beam current with a focus beam spot of $1 \mu \mathrm{m}$. Mineral and pure oxide standards were used for calibration at the same conditions followed by automatic ZAF correction and finally reported as weight percent oxides.

Nine volcanic rocks and two related dikes were subsequently selected and prepared for whole-rock geochemical analysis. Major and minor oxides were analyzed by an X-ray fluorescence spectrometer, Bruker AXS S-4 Pioneer along with rock standards (JG-2, JR-1, JG-1a, JB-1b, JA-2, GSP-2, BHVO-2, STM-1) for 

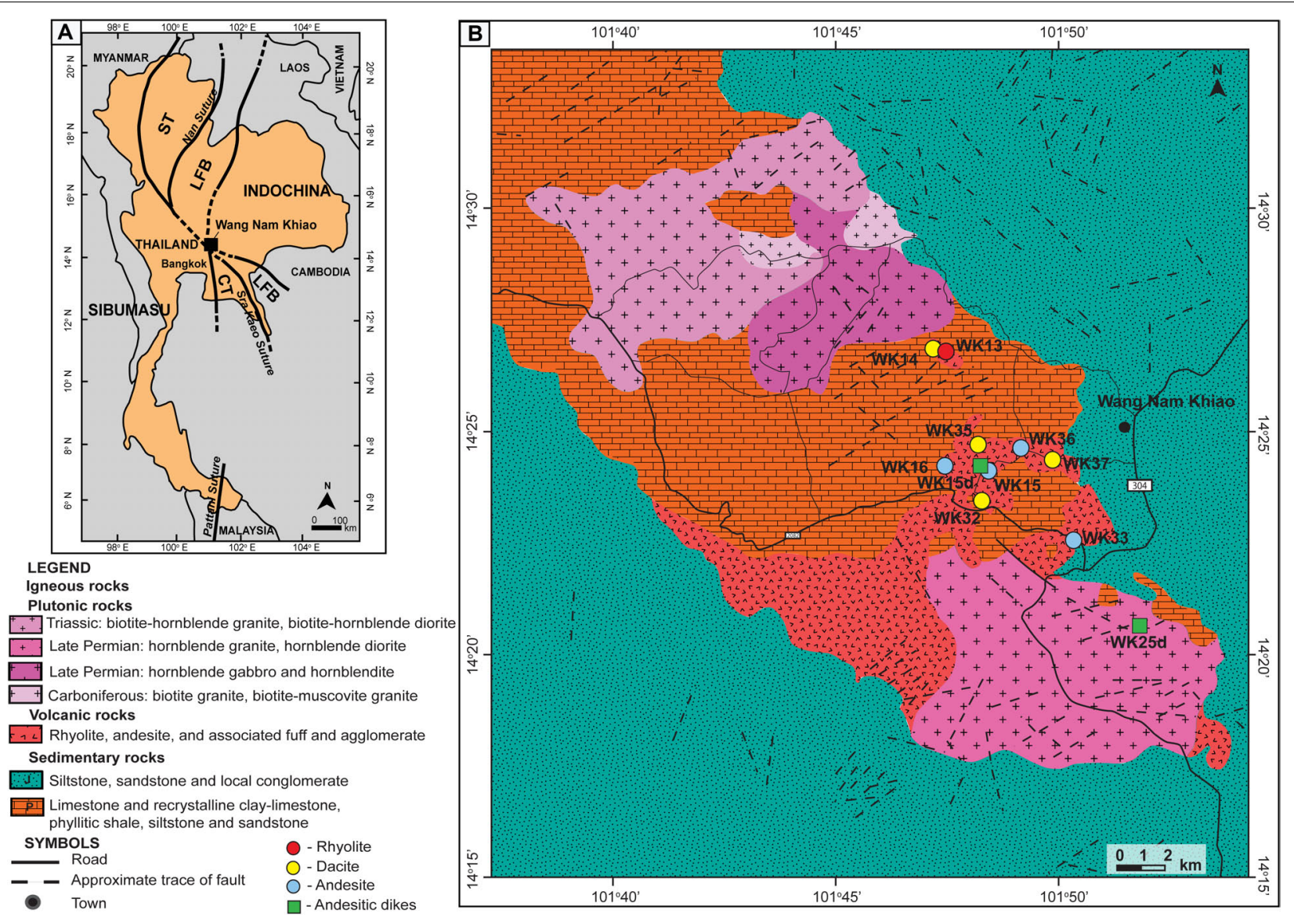

FIGURE 1 | (A) Index map of Thailand showing the study location and the main tectonic terranes including Indochina Terrane; Sibumasu (Shan-Thai) Terrane; Loei Fold Belt (LFB); Sukhothai Terrane (ST); Chanthaburi Terrane (CT) (modified from Charusiri et al., 2002; Sone and Metcalfe, 2008; Sone et al., 2012; Metcalfe, 2013; Zaw et al., 2014). (B) Geological map of the study area, Wang Nam Khiao area, Nakhon Ratchasima, and sample locations of this study (modified after Putthaphiban et al., 1989a,b; Kemlheg and Wichidchalermpong, 1992a,b).

calibration. Loss on ignition (LOI) was calculated by the weight difference of the sample after heating in a furnace at $1050^{\circ} \mathrm{C}$ for $3 \mathrm{~h}$. All research facilities reported previously are based at the Department of Geology, Faculty of Science, Chulalongkorn University.

In addition, the selected rock samples were digested using mixed hydrofluoric acid and nitric acid as suggested by Shapiro (1975) for analyses of trace and rare earth elements (REEs) using an inductively coupled plasma-mass spectrometer (ICP-MS) iCAP Q at the Scientific and Technological Research Equipment Centre, Chulalongkorn University.

\section{RESULTS}

\section{Field Survey and Petrographic Description}

Volcanic rocks cover an area of about $10 \times 12 \mathrm{~km}^{2}$ in the vicinity of Wang Nam Khiao (Figure 3A). Dikes are found cutting these volcanic rocks (Figure 3B) and the adjacent Late Permian hornblende granite (Figure 3C).
These volcanic rocks are classified as rhyolite, dacite, and andesite whereas the dikes have andesitic composition. Rhyolite and dacite are usually light gray to gray (Figure $\mathbf{3 A}$ ) and commonly show porphyritic texture. For andesite, apart from its greenish gray color resulting from enrichment of hornblende phenocrysts, the other appearances are similar to rhyolite and dacite phenocrysts. However, microscopic descriptions and geochemical analyses are meaningful for classification of these volcanic samples.

Microscopically, rhyolite and dacite similarly present abundant plagioclase phenocrysts $(\leq 1 \mathrm{~mm})$ with few hornblende phenocrysts $(\leq 0.3 \mathrm{~mm})$ embedded in fine-grained groundmass of quartz and plagioclase; moreover, their higher proportion of glass matrix (up to 30\%) exhibits obviously vitrophyric texture (Figure 3D). Plagioclase phenocrysts are typically subhedral crystal showing significant albite twins with subordinate carlsbad-albite twins whereas hornblende phenocrysts are subhedral to euhedral. Andesite shows porphyritic texture with less glass matrix in comparison with rhyolite and dacite. Moreover, it comprises abundant phenocrysts $(\leq 1 \mathrm{~mm})$ of plagioclase and hornblende with 


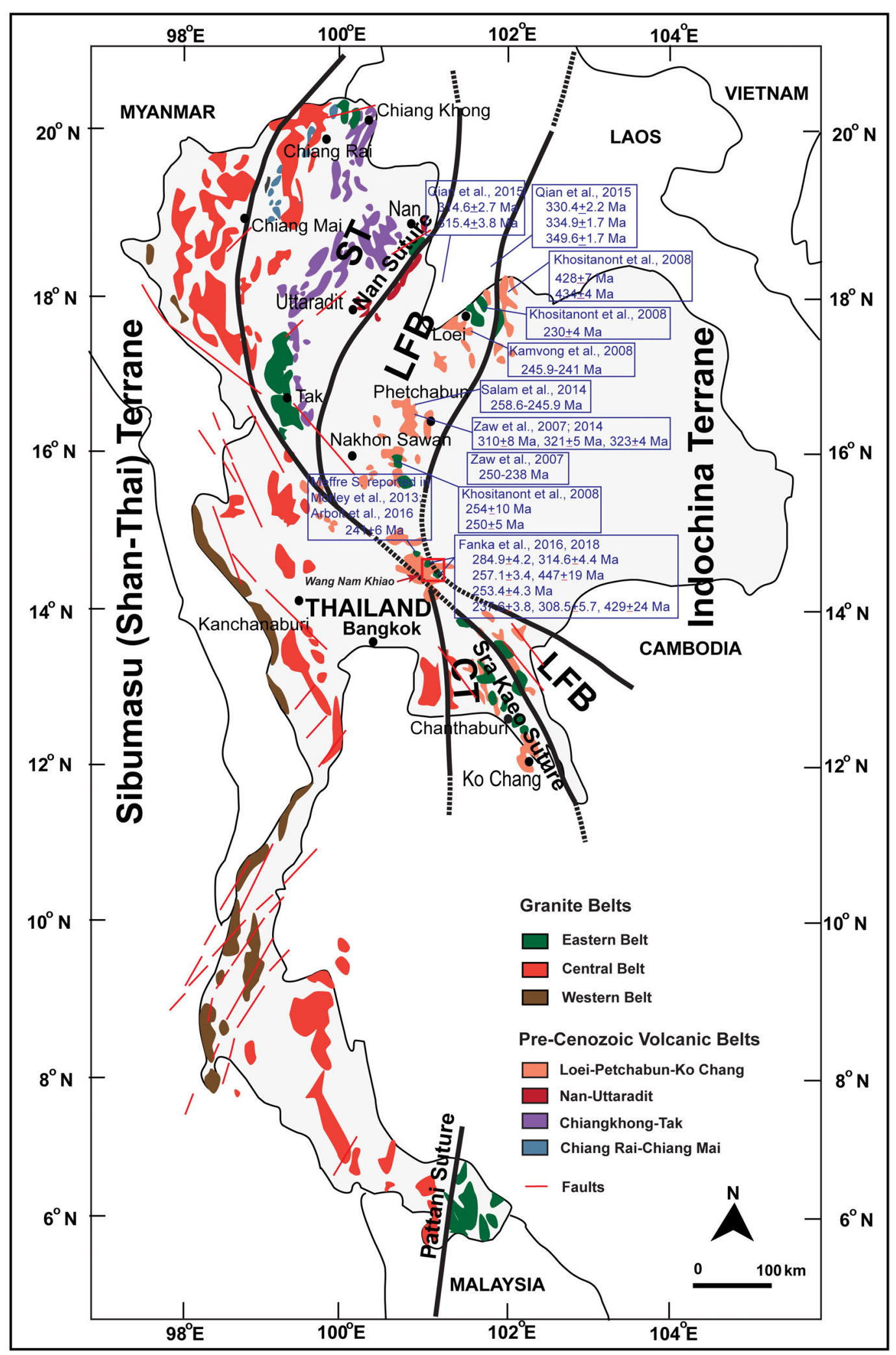

FIGURE 2 | Map shows distribution of the magmatic rocks in Thailand including the granite belts (modified after Nakapadungrat and Putthapiban, 1992) and volcanic rocks (Jungyusuk and Khositanont, 1992) together with the main tectonic terranes (modified from Charusiri et al., 2002; Sone and Metcalfe, 2008; Metcalfe, 2013; Zaw et al., 2014). Zircon U-Pb ages of the magmatic rocks in Loei Fold Belt are also present in the map. Abbreviations: LFB, Loei Fold Belt; ST, Sukhothai Terrane; CT, Chanthaburi Terrane. 

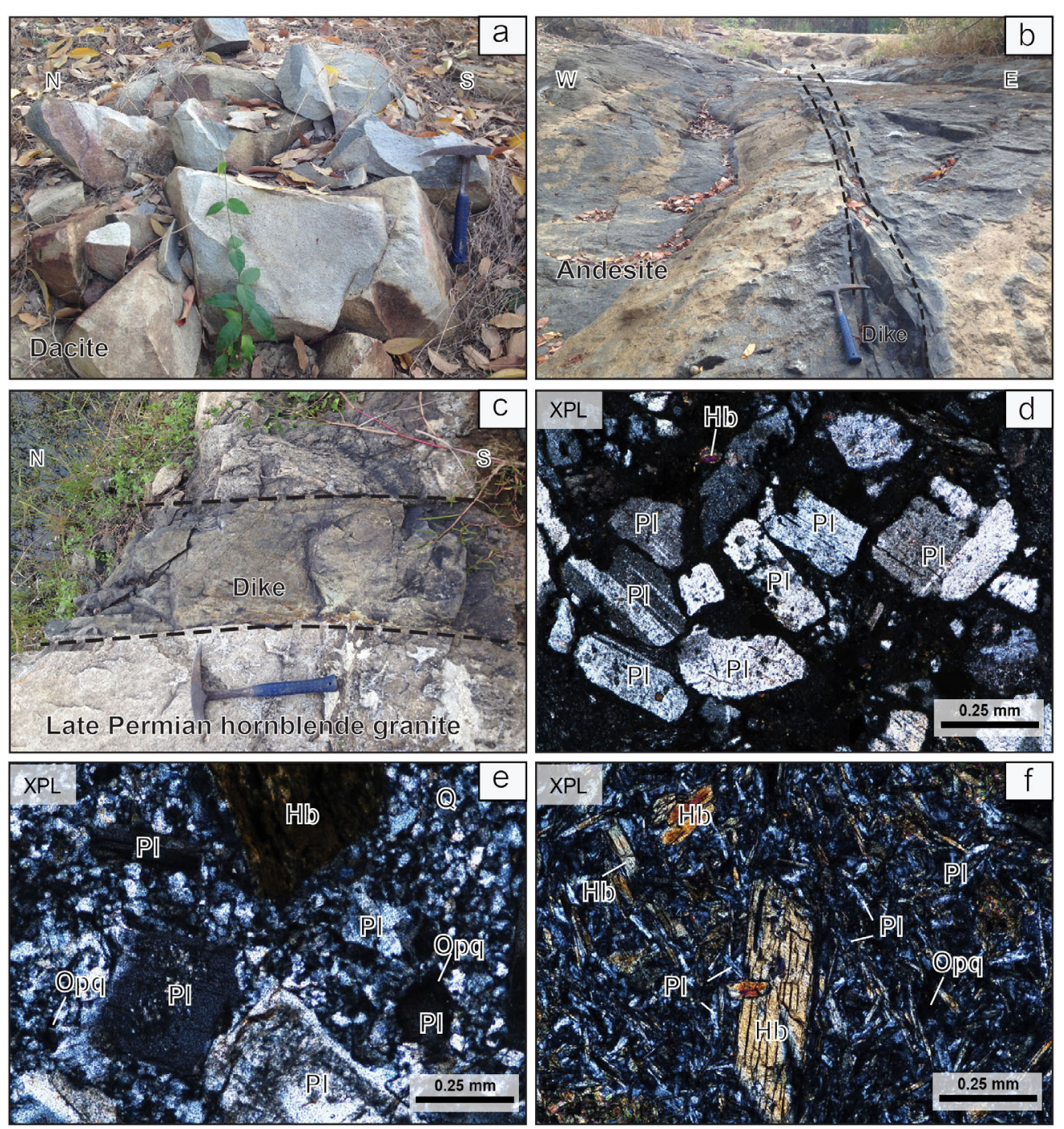

FIGURE 3 | Exposures of (a) dacite, (b) andesite cut by andesitic dike, and (c) andesitic dike cut into Late Permian hornblende granite dated by Fanka et al. (2018). Photomicrographs taken in cross-polarized light showing mineral assemblages of (d) dacite, (e) andesite, and (f) andesitic dike. Descriptions of mineral assemblage and texture are reported in the main text. Mineral abbreviations: Q, quartz; PI, plagioclase; Hb, hornblende; Opq, opaque minerals.

equal proportion (Figure 3E) surrounded by fine-grained groundmass of quartz, plagioclase, and opaque minerals. Both plagioclase and hornblende phenocrysts are mostly subhedral to euhedral.

Andesitic dikes contain more abundances of subhedraleuhedral hornblende $(\leq 1 \mathrm{~mm})$ and subhedral-euhedral plagioclase laths $(0.3-0.5 \mathrm{~mm})$ with accessory opaque minerals and glass (Figure 3F). They typically show interstitial texture with plagioclase lath and hornblende. These textures are obviously different from the volcanic samples reported previously.

\section{Mineral Chemistry}

Microprobe analyses of plagioclase and hornblende are reported in Tables 1, 2, respectively, and are illustrated in Figure 4. Compositions of mineral are effectively used to estimate pressure and temperature of crystallization, which will be reported and discussed in Section "Temperature and Pressure Estimation."

Plagioclases are abundant in all samples of volcanic and dike samples (Figures 3D-F). Plagioclase in the volcanic rocks yielded similar compositional ranges; these are 2.01-2.02 wt.\% $\mathrm{CaO}, 10.63-10.77$ wt. $\% \mathrm{Na}_{2} \mathrm{O}$, and $0.32-0.47$ wt. $\% \mathrm{~K}_{2} \mathrm{O}$ for rhyolite; 0.14-1.32 wt.\% $\mathrm{CaO}, 10.83-11.67$ wt.\% $\mathrm{Na}_{2} \mathrm{O}$, and 0.02-0.38 wt.\% $\mathrm{K}_{2} \mathrm{O}$ for dacite; and 0.87-2.11 wt.\% $\mathrm{CaO}$, 10.73-11.24 wt.\% $\mathrm{Na}_{2} \mathrm{O}$, and $0.14-0.37$ wt.\% $\mathrm{K}_{2} \mathrm{O}$ for andesite (Table 1). Moreover, they are also compositionally similar to the plagioclase of the andesitic dikes with 1.17-2.19 wt.\% $\mathrm{CaO}$, 10.43-11.12 wt.\% $\mathrm{Na}_{2} \mathrm{O}$, and 0.06-0.44 wt.\% $\mathrm{K}_{2} \mathrm{O}$ (Table 1). These plagioclases are mostly albites and few oligoclases, with the albite content ranging as $\mathrm{Ab}_{88-89}$ in the rhyolite, $\mathrm{Ab}_{92-99}$ in the dacite, $A_{89-95}$ in the andesite, and $A_{85-99}$ in the andesitic dikes (Figure 4A). 


\begin{tabular}{|c|c|c|c|c|c|c|c|c|c|c|c|c|c|c|c|c|}
\hline \multirow{2}{*}{$\begin{array}{l}\text { Rock } \\
\text { type } \\
\text { Rock } \\
\text { name }\end{array}$} & \multicolumn{2}{|c|}{ Volcanic rocks } & \multirow[b]{2}{*}{ Dacite } & \multirow[b]{2}{*}{ Dacite } & \multirow[b]{2}{*}{ Dacite } & \multirow[b]{2}{*}{ Dacite } & \multirow[b]{2}{*}{ Dacite } & \multirow[b]{2}{*}{ Dacite } & \multirow[b]{2}{*}{ Andesite } & \multirow[b]{2}{*}{ Andesite } & \multirow[b]{2}{*}{ Andesite } & \multirow{2}{*}{$\frac{\text { Dikes }}{\begin{array}{c}\text { Andesitic } \\
\text { dike }\end{array}}$} & \multirow[b]{2}{*}{$\begin{array}{l}\text { Andesitic } \\
\text { dike }\end{array}$} & \multirow[b]{2}{*}{$\begin{array}{l}\text { Andesitic } \\
\text { dike }\end{array}$} & \multirow[b]{2}{*}{$\begin{array}{l}\text { Andesitic } \\
\text { dike }\end{array}$} & \multirow[b]{2}{*}{$\begin{array}{c}\text { Andesitic } \\
\text { dike }\end{array}$} \\
\hline & Rhyolite & Rhyolite & & & & & & & & & & & & & & \\
\hline $\begin{array}{l}\text { Analysis } \\
\text { No. }\end{array}$ & $\begin{array}{l}\text { WK13 } \\
\text { PI4-1 }\end{array}$ & $\begin{array}{l}\text { WK13 } \\
\text { PI5-2 }\end{array}$ & $\begin{array}{l}\text { WK14 } \\
\text { PI1-1 }\end{array}$ & $\begin{array}{l}\text { WK14 } \\
\text { PI2-1 }\end{array}$ & $\begin{array}{l}\text { WK14 } \\
\text { PI4-2 }\end{array}$ & $\begin{array}{l}\text { WK14 } \\
\text { PI5-2 }\end{array}$ & $\begin{array}{l}\text { WK35 } \\
\text { PI5-2 }\end{array}$ & $\begin{array}{l}\text { WK35 } \\
\text { PI1-2 }\end{array}$ & $\begin{array}{l}\text { WK36 } \\
\text { PI8-2 }\end{array}$ & $\begin{array}{l}\text { WK33 } \\
\text { PI3-1 }\end{array}$ & $\begin{array}{l}\text { WK33 } \\
\text { PI1-1 }\end{array}$ & $\begin{array}{l}\text { WK15 } \\
\text { dPI1-1 }\end{array}$ & $\begin{array}{l}\text { WK15d } \\
\text { PI2-1 }\end{array}$ & $\begin{array}{l}\text { WK25d } \\
\text { PI3-1 }\end{array}$ & $\begin{array}{c}\text { WK25d } \\
\text { PI4-1 }\end{array}$ & $\begin{array}{l}\text { WK25d } \\
\text { PI4-2 }\end{array}$ \\
\hline $\mathrm{SiO}_{2}$ & 67.28 & 67.95 & 67.97 & 67.21 & 67.22 & 67.44 & 67.67 & 67.68 & 67.22 & 67.24 & 66.97 & 67.23 & 67.17 & 67.34 & 67.36 & 67.11 \\
\hline $\mathrm{Al}_{2} \mathrm{O}_{3}$ & 19.22 & 19.43 & 19.95 & 19.56 & 20.36 & 19.41 & 19.87 & 19.94 & 20.36 & 19.64 & 20.25 & 20.06 & 19.68 & 19.72 & 19.40 & 19.76 \\
\hline $\mathrm{TiO}_{2}$ & 0.00 & 0.06 & 0.00 & 0.07 & 0.00 & 0.01 & 0.00 & 0.00 & 0.00 & 0.01 & 0.01 & 0.00 & 0.00 & 0.02 & 0.01 & 0.01 \\
\hline $\mathrm{Cr}_{2} \mathrm{O}_{3}$ & 0.00 & 0.00 & 0.02 & 0.02 & 0.01 & 0.02 & 0.00 & 0.02 & 0.01 & 0.03 & 0.00 & 0.01 & 0.01 & 0.02 & 0.01 & 0.00 \\
\hline $\mathrm{FeO}$ & 0.07 & 0.06 & 0.05 & 0.19 & 0.07 & 0.15 & 0.13 & 0.19 & 0.07 & 0.08 & 0.21 & 0.07 & 0.18 & 0.17 & 0.98 & 0.09 \\
\hline $\mathrm{MnO}$ & 0.02 & 0.02 & 0.00 & 0.04 & 0.00 & 0.00 & 0.02 & 0.01 & 0.00 & 0.00 & 0.01 & 0.00 & 0.00 & 0.01 & 0.00 & 0.00 \\
\hline $\mathrm{MgO}$ & 0.00 & 0.01 & 0.00 & 0.16 & 0.01 & 0.00 & 0.00 & 0.06 & 0.01 & 0.00 & 0.00 & 0.00 & 0.04 & 0.04 & 0.67 & 0.00 \\
\hline $\mathrm{CaO}$ & 2.02 & 2.01 & 0.19 & 1.05 & 1.32 & 0.50 & 0.14 & 0.99 & 1.32 & 0.87 & 2.11 & 1.71 & 2.19 & 1.52 & 1.17 & 2.00 \\
\hline $\mathrm{Na}_{2} \mathrm{O}$ & 10.77 & 10.63 & 11.67 & 11.20 & 10.83 & 11.51 & 11.43 & 10.98 & 10.83 & 11.24 & 10.73 & 11.09 & 10.86 & 11.01 & 10.43 & 10.64 \\
\hline $\mathrm{K}_{2} \mathrm{O}$ & 0.32 & 0.47 & 0.08 & 0.02 & 0.37 & 0.05 & 0.22 & 0.38 & 0.37 & 0.16 & 0.14 & 0.06 & 0.13 & 0.36 & 0.27 & 0.13 \\
\hline Total & 99.69 & 100.65 & 99.92 & 99.52 & 100.18 & 99.09 & 99.47 & 100.25 & 100.18 & 99.28 & 100.44 & 100.23 & 100.26 & 100.21 & 100.31 & 99.72 \\
\hline \multicolumn{17}{|l|}{ 8(0) } \\
\hline $\mathrm{Si}$ & 2.967 & 2.968 & 2.974 & 2.962 & 2.944 & 2.980 & 2.976 & 2.962 & 2.944 & 2.968 & 2.933 & 2.945 & 2.948 & 2.955 & 2.955 & 2.954 \\
\hline Al & 0.999 & 1.000 & 1.028 & 1.016 & 1.051 & 1.011 & 1.029 & 1.028 & 1.051 & 1.022 & 1.045 & 1.036 & 1.017 & 1.020 & 1.003 & 1.025 \\
\hline $\mathrm{Ti}$ & 0.000 & 0.002 & 0.000 & 0.002 & 0.000 & 0.000 & 0.000 & 0.000 & 0.000 & 0.000 & 0.000 & 0.000 & 0.000 & 0.001 & 0.000 & 0.000 \\
\hline $\mathrm{Cr}$ & 0.000 & 0.000 & 0.001 & 0.001 & 0.000 & 0.001 & 0.000 & 0.001 & 0.000 & 0.001 & 0.000 & 0.000 & 0.000 & 0.001 & 0.000 & 0.000 \\
\hline $\mathrm{Fe}^{3+}$ & 0.000 & 0.000 & 0.000 & 0.000 & 0.000 & 0.000 & 0.000 & 0.000 & 0.000 & 0.000 & 0.000 & 0.000 & 0.000 & 0.000 & 0.000 & 0.000 \\
\hline $\mathrm{Fe}^{2+}$ & 0.003 & 0.002 & 0.002 & 0.007 & 0.002 & 0.005 & 0.005 & 0.007 & 0.002 & 0.003 & 0.008 & 0.003 & 0.007 & 0.006 & 0.036 & 0.003 \\
\hline $\mathrm{MnO}$ & 0.001 & 0.001 & 0.000 & 0.001 & 0.000 & 0.000 & 0.001 & 0.000 & 0.000 & 0.000 & 0.000 & 0.000 & 0.000 & 0.000 & 0.000 & 0.000 \\
\hline Mg & 0.000 & 0.001 & 0.000 & 0.011 & 0.001 & 0.000 & 0.000 & 0.004 & 0.001 & 0.000 & 0.000 & 0.000 & 0.002 & 0.002 & 0.044 & 0.000 \\
\hline $\mathrm{Ca}$ & 0.095 & 0.094 & 0.009 & 0.050 & 0.062 & 0.023 & 0.007 & 0.046 & 0.062 & 0.041 & 0.099 & 0.080 & 0.103 & 0.071 & 0.055 & 0.094 \\
\hline $\mathrm{Na}$ & 0.921 & 0.900 & 0.990 & 0.957 & 0.919 & 0.986 & 0.973 & 0.931 & 0.919 & 0.961 & 0.911 & 0.942 & 0.924 & 0.936 & 0.887 & 0.907 \\
\hline K & 0.018 & 0.026 & 0.004 & 0.001 & 0.021 & 0.003 & 0.012 & 0.021 & 0.021 & 0.009 & 0.008 & 0.003 & 0.007 & 0.020 & 0.015 & 0.007 \\
\hline Total & 5.003 & 4.993 & 5.008 & 5.007 & 5.000 & 5.009 & 5.003 & 5.000 & 5.000 & 5.006 & 5.004 & 5.009 & 5.009 & 5.013 & 4.995 & 4.991 \\
\hline An & 0.09 & 0.09 & 0.01 & 0.05 & 0.06 & 0.02 & 0.01 & 0.05 & 0.06 & 0.04 & 0.10 & 0.08 & 0.10 & 0.07 & 0.06 & 0.09 \\
\hline$A b$ & 0.89 & 0.88 & 0.99 & 0.95 & 0.92 & 0.97 & 0.98 & 0.93 & 0.92 & 0.95 & 0.89 & 0.92 & 0.89 & 0.91 & 0.93 & 0.90 \\
\hline Or & 0.02 & 0.03 & 0.00 & 0.00 & 0.02 & 0.00 & 0.01 & 0.02 & 0.02 & 0.01 & 0.01 & 0.00 & 0.01 & 0.02 & 0.02 & 0.01 \\
\hline
\end{tabular}


TABLE 2 | Representative EPMA analyses of hornblende in volcanic rocks and dikes from Wang Nam Khiao area, Nakhon Ratchasima, Thailand.

\begin{tabular}{|c|c|c|c|c|c|c|c|c|c|c|c|c|c|c|c|c|c|}
\hline \multirow{2}{*}{$\begin{array}{l}\text { Rock } \\
\text { type } \\
\text { Rock } \\
\text { name }\end{array}$} & \multicolumn{2}{|c|}{ Volcanic rocks } & \multirow[b]{2}{*}{ Rhyolite } & \multirow[b]{2}{*}{ Rhyolite } & \multirow[b]{2}{*}{ Dacite } & \multirow[b]{2}{*}{ Dacite } & \multirow[b]{2}{*}{ Dacite } & \multirow[b]{2}{*}{ Dacite } & \multirow[b]{2}{*}{ Dacite } & \multirow[b]{2}{*}{ Andesite } & \multirow[b]{2}{*}{ Andesite } & \multirow{2}{*}{$\begin{array}{c}\text { Dikes } \\
\begin{array}{c}\text { Andesitic } \\
\text { dike }\end{array}\end{array}$} & \multirow[b]{2}{*}{$\begin{array}{l}\text { Andesitic } \\
\text { dike }\end{array}$} & \multirow[b]{2}{*}{$\begin{array}{l}\text { Andesitic } \\
\text { dike }\end{array}$} & \multirow[b]{2}{*}{$\begin{array}{l}\text { Andesitic } \\
\text { dike }\end{array}$} & \multirow[b]{2}{*}{$\begin{array}{l}\text { Andesitic } \\
\text { dike }\end{array}$} & \multirow[b]{2}{*}{$\begin{array}{c}\text { Andesitic } \\
\text { dike }\end{array}$} \\
\hline & Rhyolite & Rhyolite & & & & & & & & & & & & & & & \\
\hline $\begin{array}{l}\text { Analysis } \\
\text { No. }\end{array}$ & $\begin{array}{l}\text { WK13 } \\
\text { Am2-1 }\end{array}$ & $\begin{array}{l}\text { WK13 } \\
\text { Am2-2 }\end{array}$ & $\begin{array}{l}\text { WK13 } \\
\text { Am4-2 }\end{array}$ & $\begin{array}{l}\text { WK13 } \\
\text { Am5-2 }\end{array}$ & $\begin{array}{l}\text { WK14 } \\
\text { Am2-1 }\end{array}$ & $\begin{array}{l}\text { WK14 } \\
\text { Am8-1 }\end{array}$ & $\begin{array}{l}\text { WK35 } \\
\text { Am-3 }\end{array}$ & $\begin{array}{l}\text { WK35 } \\
\text { Am-5 }\end{array}$ & $\begin{array}{l}\text { WK35 } \\
\text { Am-12 }\end{array}$ & $\begin{array}{l}\text { WK33 } \\
\text { Am1-6 }\end{array}$ & $\begin{array}{l}\text { WK33 } \\
\text { Am4-1 }\end{array}$ & $\begin{array}{r}\text { WK15d } \\
\text { Am1-2 }\end{array}$ & $\begin{array}{r}\text { WK15d } \\
\text { Am4-2 }\end{array}$ & $\begin{array}{l}\text { WK15d } \\
\text { Am14-1 }\end{array}$ & $\begin{array}{l}\text { WK15d } \\
\text { Am15-2 }\end{array}$ & $\begin{array}{l}\text { WK25d } \\
\text { Am2-2 }\end{array}$ & $\begin{array}{l}\text { WK25d } \\
\text { Am4-2 }\end{array}$ \\
\hline $\mathrm{SiO}_{2}$ & 47.34 & 47.27 & 47.34 & 47.43 & 48.07 & 48.51 & 48.40 & 48.20 & 48.43 & 48.40 & 48.10 & 47.16 & 47.24 & 47.16 & 47.01 & 47.95 & 47.44 \\
\hline $\mathrm{Al}_{2} \mathrm{O}_{3}$ & 10.52 & 10.61 & 11.13 & 10.48 & 9.94 & 9.34 & 10.44 & 10.07 & 9.35 & 9.92 & 10.54 & 10.63 & 10.03 & 10.75 & 10.45 & 11.04 & 10.66 \\
\hline $\mathrm{TiO}_{2}$ & 0.83 & 0.84 & 1.72 & 1.17 & 0.96 & 0.98 & 1.02 & 0.99 & 1.28 & 0.95 & 1.55 & 1.39 & 1.45 & 1.36 & 1.65 & 1.07 & 0.96 \\
\hline $\mathrm{Cr}_{2} \mathrm{O}_{3}$ & 0.02 & 0.01 & 0.00 & 0.01 & 0.02 & 0.03 & 0.03 & 0.00 & 0.07 & 0.03 & 0.21 & 0.05 & 0.02 & 0.01 & 0.41 & 0.00 & 0.02 \\
\hline $\mathrm{FeO}$ & 13.21 & 13.78 & 12.94 & 14.76 & 13.80 & 14.38 & 14.24 & 13.93 & 10.65 & 13.93 & 8.22 & 14.09 & 13.85 & 14.28 & 13.79 & 13.25 & 14.33 \\
\hline $\mathrm{MnO}$ & 0.34 & 0.38 & 0.08 & 0.17 & 0.27 & 0.27 & 0.24 & 0.29 & 0.16 & 0.30 & 0.11 & 0.17 & 0.15 & 0.12 & 0.16 & 0.55 & 0.54 \\
\hline $\mathrm{MgO}$ & 13.83 & 13.66 & 12.25 & 11.75 & 13.37 & 12.81 & 12.59 & 12.80 & 15.37 & 12.89 & 16.52 & 12.22 & 12.47 & 12.18 & 12.20 & 12.30 & 11.54 \\
\hline $\mathrm{CaO}$ & 10.89 & 10.71 & 10.80 & 10.80 & 10.55 & 10.49 & 10.22 & 10.40 & 11.20 & 10.69 & 10.85 & 10.21 & 11.32 & 11.11 & 11.17 & 11.14 & 10.83 \\
\hline $\mathrm{Na}_{2} \mathrm{O}$ & 1.66 & 1.85 & 2.76 & 2.45 & 1.70 & 1.72 & 1.68 & 1.75 & 1.84 & 1.76 & 2.30 & 2.00 & 2.36 & 2.27 & 2.23 & 1.97 & 1.96 \\
\hline $\mathrm{K}_{2} \mathrm{O}$ & 0.46 & 0.28 & 0.59 & 0.55 & 0.37 & 0.43 & 0.40 & 0.53 & 0.33 & 0.57 & 0.33 & 0.40 & 0.41 & 0.37 & 0.42 & 0.48 & 0.39 \\
\hline Total & 99.08 & 99.38 & 99.60 & 99.57 & 99.05 & 98.95 & 99.26 & 98.96 & 98.66 & 99.45 & 98.73 & 98.31 & 99.31 & 99.62 & 99.49 & 99.74 & 98.68 \\
\hline \multicolumn{18}{|l|}{$23(0)$} \\
\hline $\mathrm{Si}$ & 6.509 & 6.492 & 6.482 & 6.545 & 6.908 & 6.994 & 6.936 & 6.937 & 6.906 & 6.637 & 6.490 & 6.546 & 6.521 & 6.488 & 6.477 & 6.551 & 6.582 \\
\hline $\mathrm{Al}$ & 1.703 & 1.717 & 1.796 & 1.705 & 1.683 & 1.586 & 1.762 & 1.708 & 1.571 & 1.603 & 1.676 & 1.738 & 1.631 & 1.743 & 1.697 & 1.777 & 1.743 \\
\hline $\mathrm{Ti}$ & 0.085 & 0.087 & 0.177 & 0.121 & 0.104 & 0.106 & 0.110 & 0.107 & 0.137 & 0.098 & 0.157 & 0.145 & 0.151 & 0.140 & 0.171 & 0.110 & 0.101 \\
\hline $\mathrm{Cr}$ & 0.002 & 0.002 & 0.000 & 0.001 & 0.002 & 0.004 & 0.003 & 0.000 & 0.008 & 0.004 & 0.023 & 0.005 & 0.003 & 0.001 & 0.044 & 0.000 & 0.002 \\
\hline $\mathrm{Fe}^{3+}$ & 0.514 & 0.589 & 0.000 & 0.000 & 0.329 & 0.189 & 0.097 & 0.151 & 0.298 & 0.190 & 0.202 & 0.154 & 0.000 & 0.047 & 0.000 & 0.004 & 0.037 \\
\hline $\mathrm{Fe}^{2+}$ & 1.056 & 1.044 & 1.481 & 1.703 & 1.317 & 1.537 & 1.605 & 1.520 & 0.963 & 1.473 & 0.763 & 1.550 & 1.598 & 1.669 & 1.588 & 1.578 & 1.699 \\
\hline $\mathrm{Mn}$ & 0.039 & 0.044 & 0.009 & 0.020 & 0.033 & 0.032 & 0.030 & 0.035 & 0.019 & 0.035 & 0.012 & 0.020 & 0.017 & 0.014 & 0.019 & 0.063 & 0.064 \\
\hline $\mathrm{Mg}$ & 2.832 & 2.795 & 2.498 & 2.415 & 2.863 & 2.750 & 2.688 & 2.743 & 3.264 & 2.633 & 3.321 & 2.527 & 2.564 & 2.496 & 2.503 & 2.503 & 2.384 \\
\hline $\mathrm{Ca}$ & 1.603 & 1.575 & 1.583 & 1.596 & 1.624 & 1.620 & 1.568 & 1.604 & 1.710 & 1.570 & 1.568 & 1.518 & 1.674 & 1.637 & 1.648 & 1.630 & 1.609 \\
\hline $\mathrm{Na}$ & 0.443 & 0.491 & 0.731 & 0.654 & 0.473 & 0.481 & 0.466 & 0.488 & 0.508 & 0.468 & 0.602 & 0.538 & 0.631 & 0.604 & 0.596 & 0.522 & 0.528 \\
\hline K & 0.080 & 0.049 & 0.104 & 0.096 & 0.067 & 0.078 & 0.073 & 0.098 & 0.060 & 0.100 & 0.057 & 0.071 & 0.073 & 0.065 & 0.074 & 0.083 & 0.070 \\
\hline Total & 14.814 & 14.832 & 14.861 & 14.856 & 15.416 & 15.384 & 15.341 & 15.395 & 15.452 & 14.745 & 14.833 & 14.742 & 14.863 & 14.833 & 14.816 & 14.753 & 14.744 \\
\hline $\begin{array}{l}\mathrm{Mg} /(\mathrm{Fe}+ \\
\mathrm{Mg})\end{array}$ & 0.651 & 0.638 & 0.628 & 0.586 & 0.633 & 0.613 & 0.612 & 0.621 & 0.720 & 0.623 & 0.782 & 0.607 & 0.616 & 0.603 & 0.612 & 0.623 & 0.589 \\
\hline $\mathrm{Na}+\mathrm{K}$ & 0.522 & 0.540 & 0.835 & 0.750 & 0.540 & 0.559 & 0.539 & 0.586 & 0.568 & 0.568 & 0.660 & 0.609 & 0.704 & 0.669 & 0.670 & 0.605 & 0.597 \\
\hline \multicolumn{18}{|c|}{ Geobarometer (Schmidt, 1992) } \\
\hline $\begin{array}{l}\mathrm{P}(\mathrm{kbar}) \\
( \pm 0.6 \mathrm{kbar}\end{array}$ & r) 5.1 & 5.2 & 5.5 & 5.1 & 5.0 & 4.5 & 5.4 & 5.1 & 4.5 & 4.6 & 5.0 & 5.3 & 4.8 & 5.3 & 5.1 & 5.5 & 5.3 \\
\hline $\begin{array}{l}\text { Depth } \\
(\mathrm{Km})\end{array}$ & 15 & 16 & 17 & 15 & 15 & 14 & 16 & 16 & 14 & 14 & 15 & 16 & 14 & 16 & 15 & 17 & 16 \\
\hline \multicolumn{18}{|c|}{ Geothermometer (Ridolfi et al., 2010) } \\
\hline $\begin{array}{l}\mathrm{T}\left({ }^{\circ} \mathrm{C}\right) \\
\left(+57^{\circ} \mathrm{C}\right)\end{array}$ & 908 & 914 & 890 & 870 & 885 & 861 & 873 & 872 & 896 & 871 & 927 & 890 & 877 & 888 & 889 & 882 & 873 \\
\hline
\end{tabular}



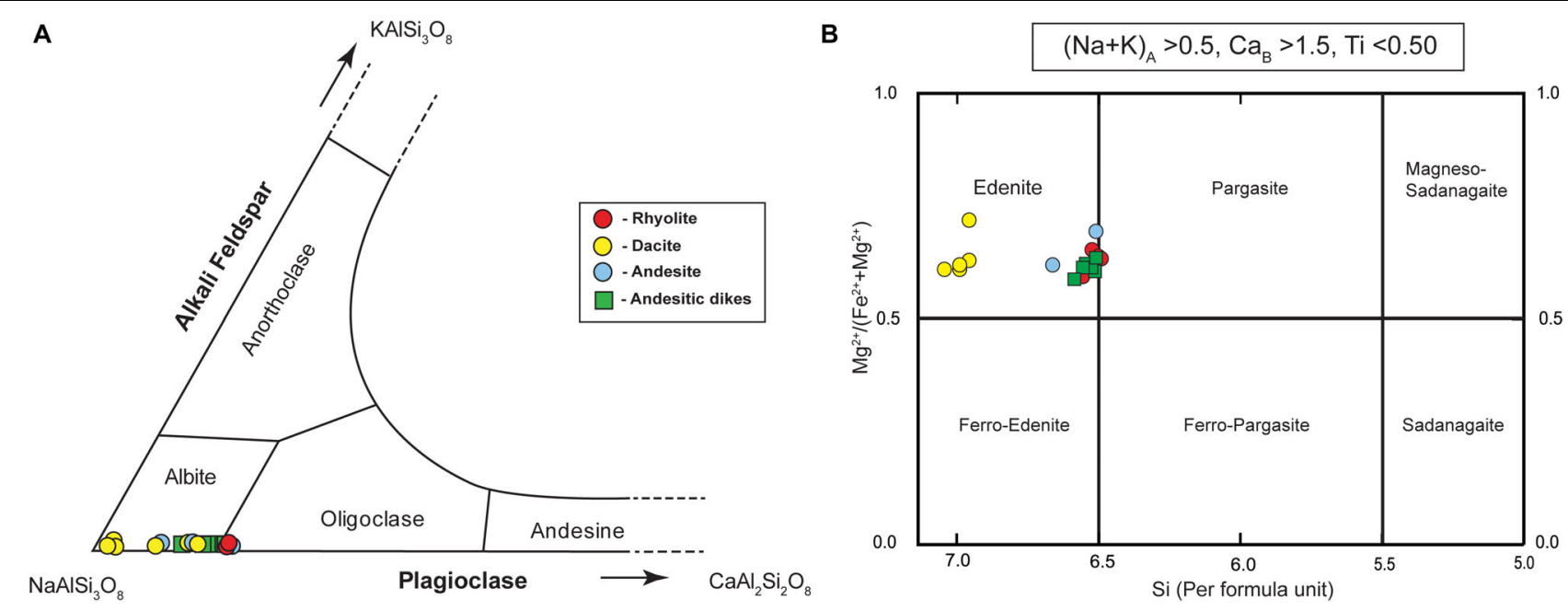

FIGURE 4 | Mineral chemical plots of (A) plagioclase (after Smith and Brown, 1974) and (B) calcic-amphibole (after Leake et al., 1997) from the volcanic rocks and related dikes in the Wang Nam Khiao area, Nakhon Ratchasima, Thailand.

Amphiboles in all volcanic samples and andesitic dikes yielded similar compositional ranges with 47.01-48.51 wt.\% $\mathrm{SiO}_{2}, 9.34-$ 11.13 wt.\% $\mathrm{Al}_{2} \mathrm{O}_{3}, 8.22-14.76$ wt.\% $\mathrm{FeO}_{\mathrm{t}}, 11.54-16.52$ wt.\% $\mathrm{MgO}$, and 10.21-11.32 wt.\% CaO. According to the international classification scheme, the analyzed amphiboles from all samples are classified as edenites (Figure 4B).

\section{Whole-Rock Geochemistry}

Major and minor compositions of the representative rhyolite, dacite, andesite, and related andesitic dikes in the Wang Nam Khiao area, Nakhon Ratchasima, Thailand, are shown in Table 3. These volcanic rocks and related dikes show slightly different major and minor compositions.

$\mathrm{SiO}_{2}$ contents are expectedly higher in the rhyolite and lower in the andesite. The other major oxides of all volcanic rocks include 11.33-15.30 wt.\% $\mathrm{Al}_{2} \mathrm{O}_{3}, 1.90-10.27$ wt.\% $\mathrm{FeO}_{\mathrm{t}}, 2.64-7.53$ wt.\% $\mathrm{CaO}, 3.20-5.29$ wt.\% $\mathrm{Na}_{2} \mathrm{O}$, and 1.882.54 wt.\% $\mathrm{K}_{2} \mathrm{O}$. Andesitic dikes present 54.60-59.78 wt.\% $\mathrm{SiO}_{2}, \quad 11.74-13.02$ wt.\% $\mathrm{Al}_{2} \mathrm{O}_{3}, \quad 6.87-8.21$ wt.\% $\mathrm{FeO}_{\mathrm{t}}$, 4.91-6.83 wt.\% $\mathrm{CaO}$, and 3.33-3.40 wt.\% $\mathrm{Na}_{2} \mathrm{O}$. These andesitic dikes display slightly higher $\mathrm{MgO}$ content (8.3810.35 wt.\%) than those of volcanic rocks (3.47-6.70 wt.\% for andesite, 2.61-9.21 wt.\% for dacite, and 1.52 wt.\% for rhyolite). $\mathrm{Na}_{2} \mathrm{O}+\mathrm{K}_{2} \mathrm{O}$ contents of dikes (4.70-5.55 wt.\%) are similar to those of andesite (3.55-6.07 wt.\%), which are lower than those of dacite (4.85-6.67 wt.\%) and rhyolite (7.30 wt.\%), respectively.

On a $\mathrm{Nb} / \mathrm{Y}$ versus $\mathrm{Zr} / \mathrm{Ti}$ diagram (Winchester and Floyd, 1977) (Figure 5), the analyzed samples plot in the fields of rhyolite, dacite, and andesite, accordingly. Harker variation diagrams (Harker, 1909) show negative correlation of $\mathrm{SiO}_{2}$ against $\mathrm{TiO}_{2}, \mathrm{CaO}, \mathrm{FeO}$, and $\mathrm{MgO}$ and positive correlations of $\mathrm{SiO}_{2}$ versus $\mathrm{Na}_{2} \mathrm{O}$ and $\mathrm{K}_{2} \mathrm{O}$ (Figure 6). These chemical charters are compatible with their mineral assemblages, especially increasing proportions of albite and quartz and decreasing proportion of hornblende from the andesite toward dacite and rhyolite, respectively. The linear correlations indicate that these rocks are related through a single magmatic differentiation.

Primitive mantle-normalized spider diagrams (Figure 7A) of most rock samples reveal almost fractionation patterns from the large-ion lithophile elements (LILE) to high-field-strength elements (HFSE) with slightly positive anomalies of $\mathrm{Rb}, \mathrm{K}$, and $\mathrm{Sr}$ and negative anomalies of $\mathrm{Nb}, \mathrm{Ce}$, and $\mathrm{Ti}$. In addition, the chondrite-normalized REE patterns of all rock types are slightly high in light REE (LREE) when data of chondrite from Sun and McDonough (1989) were used for normalization. Regarding chondrite-normalized $\mathrm{La} / \mathrm{Yb},(\mathrm{La} / \mathrm{Yb})_{\mathrm{N}}$ ratios of dike samples (8.64-9.41) are in the range of volcanic rocks (6.05-14.54 for andesite, 10.77-21.86 for dacite, and 10.77 for rhyolite). In terms of $(\mathrm{La} / \mathrm{Sm})_{\mathrm{N}}$ ratios, volcanic rocks are 2.58 in the rhyolite, 2.58-5.89 in the dacite, and 2.35-3.06 in the andesite whereas andesitic dikes are 2.57-2.93. Moreover, the primitive mantle-normalized spider diagrams and chondritenormalized REE patterns are comparable with the typical volcanic arc setting reported by Blein et al. (2001) and Reubi and Nicholls (2004); however, the samples' patterns present slightly lower HREE than those typical volcanic arc patterns (Figures 7A,B).

\section{DISCUSSION}

\section{Temperature and Pressure Estimation}

Microprobe data have been used to estimate pressure and temperature of crystallization in volcanic rocks as suggested by Kuprubasi et al. (2014). Although some mineral textures (e.g., zoned texture, corona texture, reaction rim, alteration) in volcanic rocks are not in equilibrium, the undisturbed single and/or coexisting minerals as phenocrysts can be used to estimate the $\mathrm{P}-\mathrm{T}$ condition of crystallization (Foden and 
TABLE 3 | Representative whole-rock geochemistry of volcanic rocks and related dikes from the Wang Nam Khiao area, Nakhon Ratchasima, Thailand (major and minor oxides in wt\%, trace elements and REE in ppm).

\begin{tabular}{|c|c|c|c|c|c|c|c|c|c|c|c|}
\hline \multirow{2}{*}{$\begin{array}{l}\text { Rock type: } \\
\text { Rock name: }\end{array}$} & \multicolumn{2}{|c|}{ Volcanic rocks } & \multirow[b]{2}{*}{ Dacite } & \multirow[b]{2}{*}{ Dacite } & \multirow[b]{2}{*}{ Dacite } & \multirow[b]{2}{*}{ Andesite } & \multirow[b]{2}{*}{ Andesite } & \multirow[b]{2}{*}{ Andesite } & \multirow[b]{2}{*}{ Andesite } & \multirow{2}{*}{$\frac{\text { Dikes }}{\text { Andesitic dike }}$} & \multirow[b]{2}{*}{ Andesitic dike } \\
\hline & Rhyolite & Dacite & & & & & & & & & \\
\hline Sample no: & $3 W K 13$ & $3 W K 14$ & $3 W K 32$ & 3WK35 & 3WK37 & $3 W K 15$ & $3 W K 16$ & 3WK33 & $3 W K 36$ & $3 W K 15 d$ & $3 W K 25 d$ \\
\hline \multicolumn{12}{|c|}{ Major and minor oxide (\%) } \\
\hline $\mathrm{SiO}_{2}$ & 68.27 & 66.62 & 62.32 & 58.62 & 63.95 & 63.91 & 63.82 & 56.35 & 56.90 & 54.60 & 59.78 \\
\hline $\mathrm{TiO}_{2}$ & 0.25 & 0.33 & 0.57 & 0.56 & 0.58 & 0.55 & 0.58 & 1.08 & 0.95 & 0.69 & 0.69 \\
\hline $\mathrm{Al}_{2} \mathrm{O}_{3}$ & 15.30 & 14.71 & 14.70 & 11.33 & 14.64 & 14.89 & 14.72 & 12.76 & 14.62 & 13.02 & 11.74 \\
\hline $\mathrm{FeOt}$ & 1.90 & 2.71 & 6.11 & 7.74 & 5.74 & 5.24 & 5.56 & 10.27 & 9.49 & 8.21 & 6.87 \\
\hline $\mathrm{MnO}$ & 0.00 & 0.00 & 0.00 & 0.11 & 0.00 & 0.00 & 0.09 & 0.15 & 0.19 & 0.13 & 0.11 \\
\hline $\mathrm{MgO}$ & 1.52 & 2.61 & 4.60 & 9.21 & 4.29 & 4.58 & 3.47 & 6.70 & 6.36 & 10.35 & 8.38 \\
\hline $\mathrm{CaO}$ & 2.64 & 3.19 & 3.96 & 5.53 & 3.45 & 3.39 & 4.48 & 7.53 & 4.15 & 6.83 & 4.91 \\
\hline $\mathrm{Na}_{2} \mathrm{O}$ & 5.29 & 4.62 & 3.98 & 3.25 & 4.33 & 3.53 & 3.48 & 3.20 & 5.15 & 3.33 & 3.40 \\
\hline $\mathrm{K}_{2} \mathrm{O}$ & 2.01 & 2.05 & 1.76 & 1.59 & 1.88 & 2.54 & 2.10 & 0.35 & 0.61 & 1.37 & 2.15 \\
\hline $\mathrm{P}_{2} \mathrm{O}_{5}$ & 0.05 & 0.13 & 0.22 & 0.17 & 0.27 & 0.22 & 0.25 & 0.31 & 0.29 & 0.03 & 0.17 \\
\hline LOI & 0.96 & 0.78 & 2.04 & 1.02 & 1.11 & 1.03 & 1.23 & 1.02 & 1.09 & 1.07 & 1.17 \\
\hline Total & 98.20 & 97.75 & 100.24 & 99.15 & 100.24 & 99.90 & 99.79 & 99.72 & 99.81 & 99.63 & 99.37 \\
\hline \multicolumn{12}{|c|}{ Trace elements and REE (ppm) } \\
\hline $\mathrm{Li}$ & 11.82 & 10.64 & 7.81 & 10.85 & 9.68 & 24.03 & 24.09 & 4.95 & 12.32 & 20.78 & 15.81 \\
\hline $\mathrm{Be}$ & 1.48 & 1.62 & 1.15 & 0.69 & 1.02 & 2.04 & 1.46 & 0.57 & 0.80 & 0.93 & 0.84 \\
\hline V & 35.59 & 61.62 & 105.78 & 128.13 & 97.27 & 121.98 & 96.09 & 237.98 & 134.84 & 174.72 & 134.53 \\
\hline $\mathrm{Cr}$ & 20.68 & 67.25 & 93.66 & 393.91 & 41.43 & 76.58 & 64.04 & 10.60 & 19.23 & 387.42 & 214.34 \\
\hline Sc & 3.64 & 6.77 & 12.17 & 16.15 & 9.08 & 14.36 & 11.80 & 18.95 & 17.11 & 21.74 & 15.11 \\
\hline $\mathrm{Ni}$ & 7.83 & 23.96 & 51.11 & 133.27 & 20.16 & 36.74 & 28.57 & 8.17 & 13.62 & 128.31 & 111.12 \\
\hline $\mathrm{Cu}$ & 9.68 & 7.92 & 34.95 & 65.60 & 15.80 & 26.64 & 26.06 & 137.20 & 36.97 & 57.33 & 39.69 \\
\hline Mn & 243.08 & 312.88 & 532.63 & 719.38 & 527.90 & 876.93 & 721.24 & 1008.48 & 1151.17 & 1016.23 & 720.76 \\
\hline As & 0.88 & 0.98 & 3.76 & 5.30 & 4.40 & 1.69 & 1.56 & 0.84 & 2.02 & 1.01 & 4.36 \\
\hline Co & 50.96 & 48.97 & 32.99 & 37.28 & 27.71 & 58.67 & 54.33 & 29.67 & 20.19 & 43.76 & 31.27 \\
\hline Zn & 46.98 & 41.72 & 54.54 & 48.98 & 40.91 & 65.83 & 52.29 & 67.16 & 63.11 & 60.36 & 59.03 \\
\hline $\mathrm{Ga}$ & 20.48 & 21.58 & 16.86 & 13.86 & 15.87 & 21.51 & 16.97 & 14.74 & 15.35 & 17.13 & 15.76 \\
\hline $\mathrm{Rb}$ & 52.88 & 54.78 & 30.36 & 26.58 & 24.38 & 152.63 & 74.54 & 7.87 & 13.54 & 40.16 & 49.65 \\
\hline $\mathrm{Sr}$ & 1068.30 & 1251.95 & 1092.19 & 937.33 & 1169.15 & 413.24 & 475.14 & 491.45 & 487.44 & 723.19 & 739.96 \\
\hline Y & 5.88 & 9.06 & 18.56 & 12.88 & 12.56 & 18.29 & 14.96 & 17.35 & 18.90 & 13.64 & 11.97 \\
\hline $\mathrm{Zr}$ & 96.39 & 95.59 & 103.91 & 99.82 & 106.30 & 88.00 & 88.21 & 99.28 & 96.10 & 106.11 & 109.30 \\
\hline Sn & 2.56 & 0.85 & 1.14 & 0.89 & 0.87 & 1.92 & 1.63 & 1.70 & 0.88 & 1.21 & 1.04 \\
\hline $\mathrm{Sb}$ & 0.26 & 0.23 & 0.25 & 0.54 & 0.43 & 0.25 & 0.42 & 0.17 & 0.64 & 0.25 & 0.33 \\
\hline Cs & 0.97 & 0.93 & 0.82 & 2.86 & 1.02 & 5.18 & 2.63 & 0.24 & 0.61 & 1.54 & 0.27 \\
\hline $\mathrm{Ba}$ & 284.20 & 225.88 & 62.29 & 43.30 & 251.71 & 386.30 & 348.78 & 208.39 & 123.46 & 278.57 & 173.05 \\
\hline La & 6.92 & 9.03 & 27.67 & 9.69 & 19.37 & 38.43 & 35.22 & 11.27 & 13.64 & 13.60 & 11.15 \\
\hline $\mathrm{Ce}$ & 14.59 & 19.76 & 46.54 & 17.85 & 38.69 & 68.59 & 63.64 & 21.42 & 28.24 & 27.10 & 23.91 \\
\hline $\operatorname{Pr}$ & 1.76 & 2.38 & 7.22 & 2.72 & 4.77 & 6.42 & 6.48 & 2.78 & 3.57 & 3.37 & 3.08 \\
\hline $\mathrm{Nd}$ & 8.07 & 10.89 & 29.67 & 12.20 & 19.92 & 24.35 & 22.65 & 12.40 & 16.13 & 14.40 & 13.18 \\
\hline $\mathrm{Sm}$ & 1.73 & 2.25 & 5.84 & 2.63 & 3.84 & 4.25 & 3.86 & 3.00 & 3.75 & 3.00 & 2.80 \\
\hline $\mathrm{Gd}$ & 1.19 & 1.57 & 4.01 & 2.02 & 2.62 & 3.27 & 3.01 & 2.49 & 3.02 & 2.23 & 2.09 \\
\hline $\mathrm{Tb}$ & 0.18 & 0.25 & 0.63 & 0.35 & 0.39 & 0.49 & 0.45 & 0.45 & 0.52 & 0.37 & 0.35 \\
\hline Dy & 0.88 & 1.23 & 3.04 & 1.85 & 1.93 & 2.47 & 2.26 & 2.51 & 2.85 & 1.92 & 1.80 \\
\hline Ho & 0.12 & 0.18 & 0.43 & 0.29 & 0.29 & 0.36 & 0.34 & 0.39 & 0.43 & 0.29 & 0.27 \\
\hline Er & 0.41 & 0.60 & 1.43 & 0.96 & 0.99 & 1.24 & 1.16 & 1.33 & 1.42 & 1.01 & 0.91 \\
\hline Tm & 0.06 & 0.09 & 0.22 & 0.15 & 0.16 & 0.20 & 0.18 & 0.21 & 0.22 & 0.16 & 0.15 \\
\hline $\mathrm{Yb}$ & 0.41 & 0.60 & 1.37 & 0.95 & 1.02 & 1.27 & 1.16 & 1.34 & 1.32 & 1.04 & 0.93 \\
\hline Lu & 0.06 & 0.09 & 0.21 & 0.16 & 0.17 & 0.20 & 0.18 & 0.22 & 0.20 & 0.17 & 0.15 \\
\hline $\mathrm{Nb}$ & 3.49 & 2.26 & 5.19 & 3.40 & 8.09 & 26.19 & 20.08 & 5.21 & 6.84 & 7.79 & 7.66 \\
\hline
\end{tabular}


TABLE 3 | Continued

\begin{tabular}{|c|c|c|c|c|c|c|c|c|c|c|c|}
\hline \multirow{2}{*}{$\begin{array}{l}\text { Rock type: } \\
\text { Rock name: }\end{array}$} & \multicolumn{2}{|c|}{ Volcanic rocks } & \multirow[b]{2}{*}{ Dacite } & \multirow[b]{2}{*}{ Dacite } & \multirow[b]{2}{*}{ Dacite } & \multirow[b]{2}{*}{ Andesite } & \multirow[b]{2}{*}{ Andesite } & \multirow[b]{2}{*}{ Andesite } & \multirow[b]{2}{*}{ Andesite } & \multirow{2}{*}{$\begin{array}{c}\text { Dikes } \\
\text { Andesitic dike }\end{array}$} & \multirow[b]{2}{*}{ Andesitic dike } \\
\hline & Rhyolite & Dacite & & & & & & & & & \\
\hline Sample no: & $3 W K 13$ & $3 W K 14$ & $3 W K 32$ & 3WK35 & 3WK37 & $3 W K 15$ & $3 W K 16$ & 3WK33 & 3WK36 & $3 W K 15 d$ & $3 W K 25 d$ \\
\hline $\mathrm{Ta}$ & 2.60 & 0.88 & 1.72 & 1.58 & 4.78 & 6.04 & 3.03 & 2.05 & 1.40 & 3.15 & 2.62 \\
\hline $\mathrm{Pb}$ & 9.06 & 5.89 & 6.50 & 10.31 & 8.97 & 12.70 & 12.01 & 3.96 & 4.40 & 5.31 & 6.69 \\
\hline $\mathrm{Bi}$ & 0.02 & 0.03 & 0.03 & 2.01 & 0.05 & 0.36 & 0.64 & 0.02 & 0.04 & 0.03 & 0.10 \\
\hline Th & 1.14 & 1.33 & 2.23 & 1.26 & 4.03 & 13.49 & 15.34 & 2.01 & 2.55 & 2.24 & 2.56 \\
\hline$U$ & 0.80 & 1.04 & 1.01 & 0.62 & 1.58 & 3.94 & 2.24 & 0.60 & 0.80 & 0.79 & 1.29 \\
\hline Mo & 0.67 & 1.33 & 0.49 & 0.44 & 0.45 & 0.92 & 0.60 & 0.67 & 0.68 & 0.58 & 0.46 \\
\hline $\mathrm{Eu}$ & 0.52 & 0.69 & 1.63 & 0.86 & 1.09 & 1.24 & 1.00 & 1.15 & 1.47 & 0.92 & 0.92 \\
\hline $\mathrm{Hf}$ & 1.97 & 1.53 & 1.98 & 1.59 & 2.36 & 0.68 & 0.50 & 1.42 & 1.28 & 2.08 & 2.39 \\
\hline $\operatorname{Re}$ & 0.01 & 0.01 & 0.00 & 0.00 & 0.00 & 0.01 & 0.01 & 0.00 & 0.00 & 0.00 & 0.00 \\
\hline
\end{tabular}

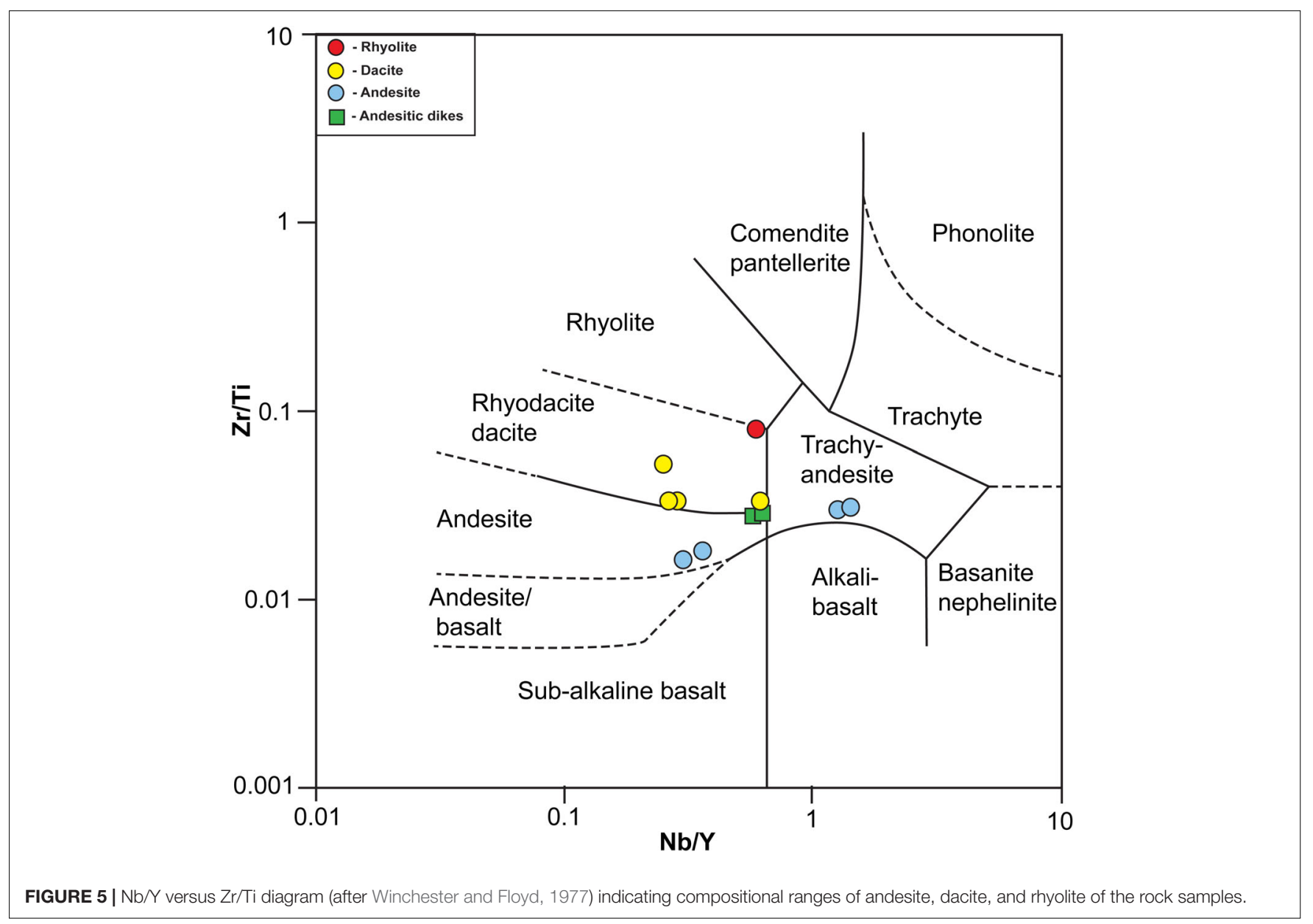

Green, 1992; Kuprubasi et al., 2014). Therefore, mineral compositions of undisturbed phenocrysts were used for the calculation of P-T conditions. Plagioclase compositions are sensitive to melt-water concentration (Lange et al., 2009) and alteration processes (Zhu and Lu, 2009; Hovelmann et al., 2010; Yuan et al., 2019); therefore, hornblende is efficiently used to calculate the P-T conditions (Schmidt, 1992; Ridolfi et al., 2010; Ridolfi and Renzulli, 2012). Al-in-hornblende geobarometer and hornblende geothermometer are employed to calculate the $\mathrm{P}-\mathrm{T}$ conditions of crystallization in this study.

Ridolfi et al. (2010) proposed a hornblende geothermometer for the calc-alkaline volcanic system related to subduction based on the experimental data and natural data from worldwide calc-alkaline volcanoes, with the uncertain calibration of $\pm 57^{\circ} \mathrm{C}$ by using the Silicon index $\left(\mathrm{Si}^{*}\right)$ of hornblende composition 

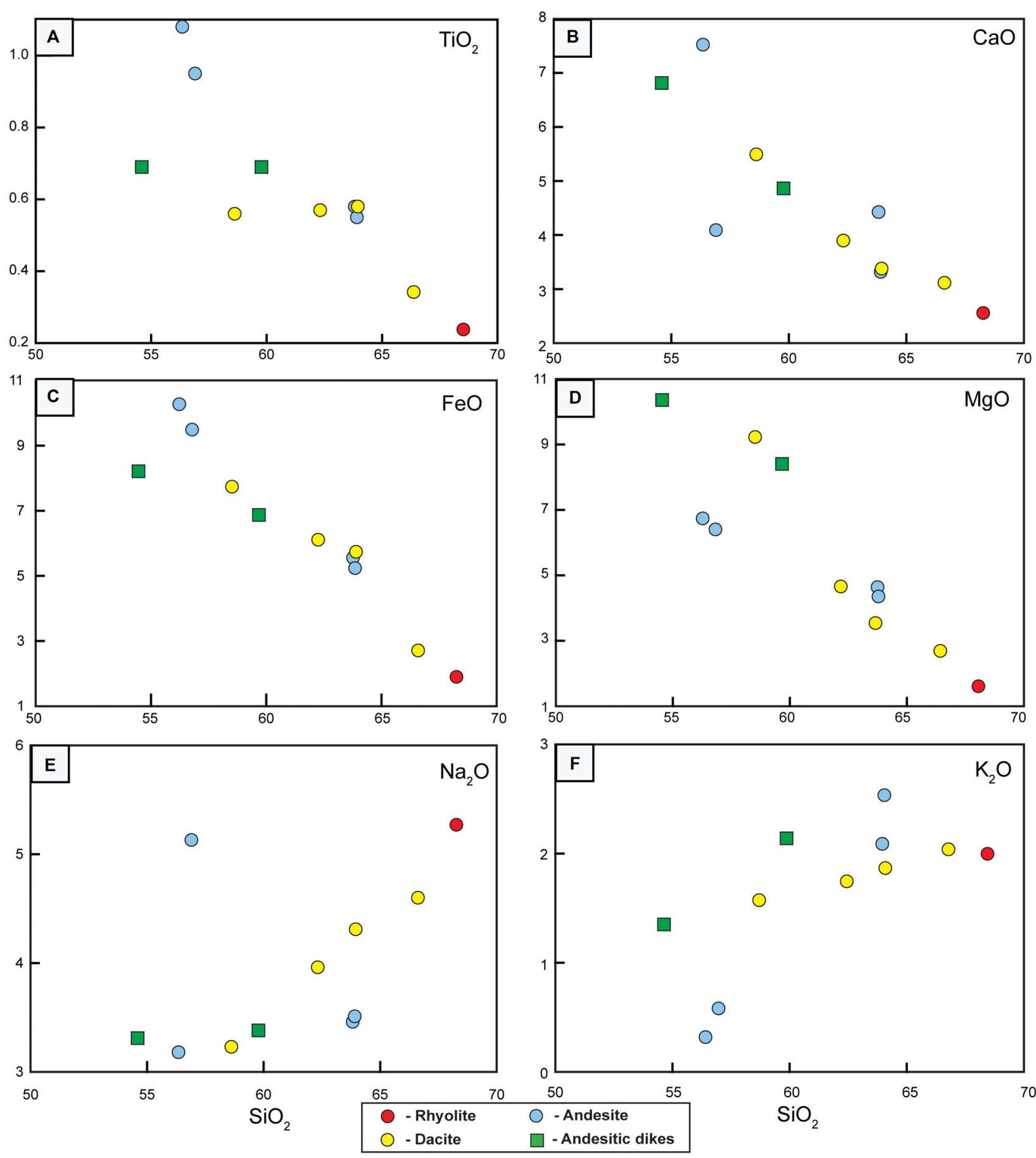

FIGURE 6 | Variation diagrams (Harker, 1909) of $\mathrm{SiO}_{2}$ against other major and minor oxides for (A-D) negative and (E-F) positive correlation of volcanic rocks and dikes in Wang Nam Khiao area, Nakhon Ratchasima, Thailand.

in the equation: $\mathrm{T}\left({ }^{\circ} \mathrm{C}\right)=-151.487 \mathrm{Si}^{*}+2041$, where $\mathrm{T}$ is temperature and $\mathrm{Si}^{*}=\mathrm{Si}+\left({ }^{[4]} \mathrm{Al} / 15\right)-\left({ }^{[4]} \mathrm{Ti}\right)-\left({ }^{[6]} \mathrm{Al} / 2\right)-$ $\left({ }^{[6]} \mathrm{Ti} / 1.8\right)+\left(\mathrm{Fe}^{3+} / 9\right)+\left(\mathrm{Fe}^{2+} / 3.3\right)+(\mathrm{Mg} / 26)+\left({ }^{\mathrm{B}} \mathrm{Ca} / 5\right)+$ $\left({ }^{\mathrm{B}} \mathrm{Na} / 1.3\right)-\left({ }^{\mathrm{A}} \mathrm{Na} / 15\right)+\left({ }^{\mathrm{A}}[\mathrm{Na}+\mathrm{K}] / 2.3\right)$. Based on the hornblende geothermometry, calculated temperatures of the representative volcanic rocks vary from 861 to $927^{\circ} \mathrm{C}$ whereas the dike samples yield about 873 to $890^{\circ} \mathrm{C}$ (Table 2). These estimated temperatures are comparable with the crystallization temperature range of calibrated geothermometer for andesite-rhyolite in the calc-alkaline volcanic system (Ridolfi et al., 2010; Walker et al., 2013; Waters and Lange, 2015; Chen et al., 2017). 

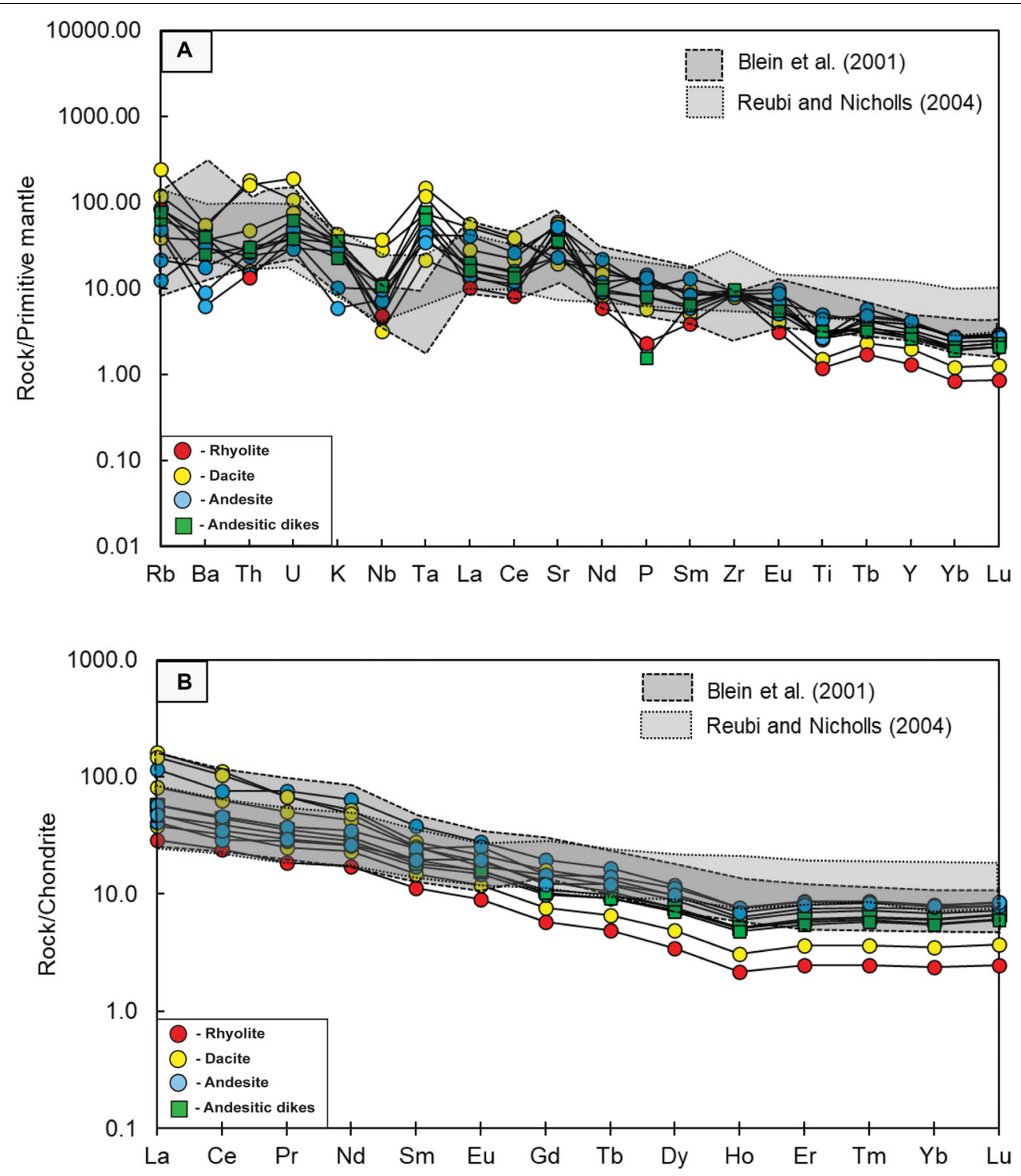

FIGURE 7 | (A) Primitive mantle-normalized spider diagrams (primitive mantle values from Sun and McDonough, 1989) and (B) chondrite-normalized REE patterns (chondrite values from Sun and McDonough, 1989) of the studied rocks and shade patterns volcanic rocks from typical arc setting (data from Blein et al., 2001; Jicha et al., 2004; Reubi and Nicholls, 2004; Jicha and Singer, 2006) for comparison.

The Al-in-hornblende geobarometry has been widely used to calculate the pressure of magmatic crystallization (e.g., Hammarstrom and Zen, 1986; Schmidt, 1992; Helmy et al., 2004; Hossain et al., 2009) using the aluminum partition in hornblende, which was provided by experimental studies (Hollister et al., 1987; Johnson and Rutherford, 1989; Schmidt, 1992; Anderson and Smith, 1995). In this study, the calibration equation: Al-in-hornblende geobarometry $\mathrm{P}(\mathrm{kbar})=4.76$ $\mathrm{Al}^{\text {tot }}-3.01$ with error $\pm 0.6 \mathrm{kbar}$ of Schmidt (1992) in the $\mathrm{H}_{2} \mathrm{O}$-saturated condition was employed to calculate the pressure of magmatic crystallization. The calculated results are presented in Table 2. The calculated pressures of the volcanic rocks and related dikes fall in similar ranges of 4.55.5 and $4.8-5.5 \mathrm{kbar}$, respectively. As the calculated pressure from hornblende geobarometer has been used to indicate the depths of magma reservoir beneath arc volcanoes (Scott et al., 2012; Costa et al., 2013; Rivera et al., 2017), these calculated pressures can indicate the crystallization pressures of the phenocrysts from a magma reservoir that extruded to the surface.

Moreover, the pressures calculated from hornblende phenocrysts are used to estimate the depth by using the geobarometric gradient of $0.33 \mathrm{kbar} / \mathrm{km}$ (Bethel-Thompson et al., 2014). As a result, depths of crystallization are estimated at 14-17 km for the magma intrusion that fed volcanic rocks and dikes (Table 2). The depths should indicate the crystallization depths of the phenocrysts before complete crystallization of volcanic rocks at the shallower up to the surface. These 


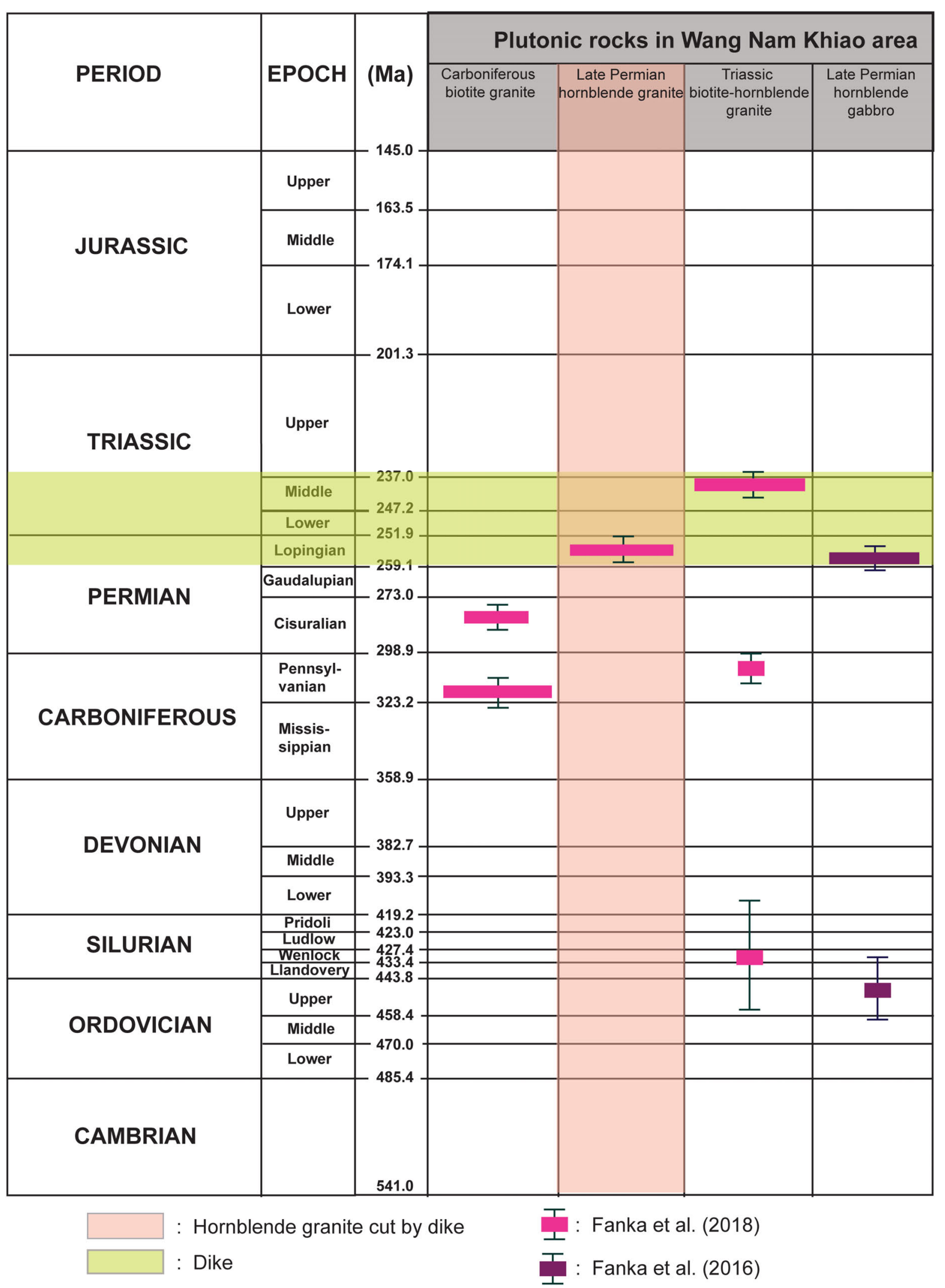

FIGURE 8 | Zircon U-Pb ages of plutonic rocks and the estimated age of the dike cut into the Late Permian hornblende granite exposed in the Wang Nam Khiao area, Nakhon Ratchasima, Thailand (modified from Fanka et al., 2018). 


\section{A Late Carboniferous - Early Permian}

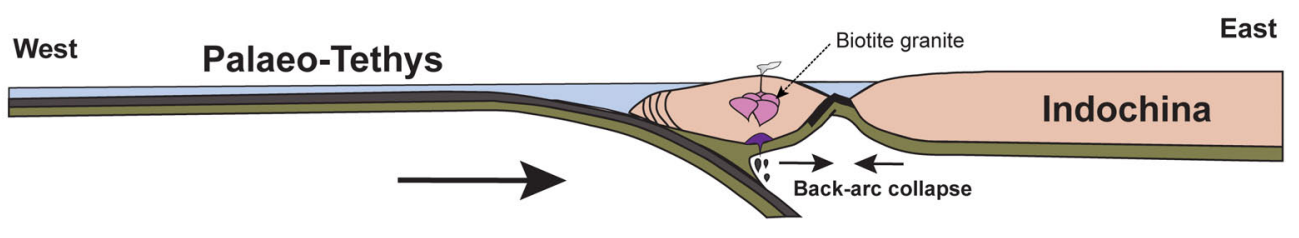

\section{B Late Permian}

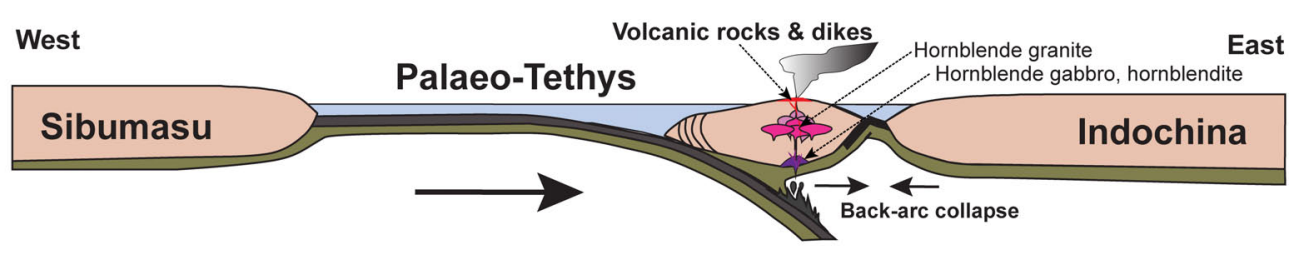

\section{c Middle Triassic}

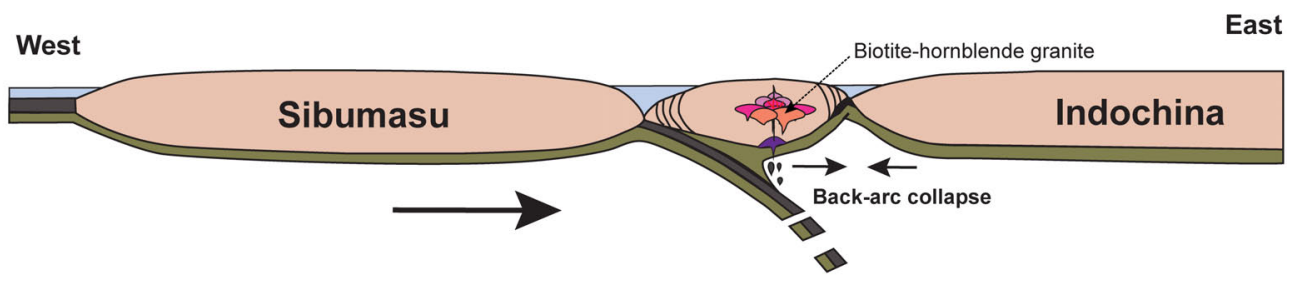

FIGURE 9 | Schematic tectonic evolution model and arc magmatism in the Wang Nam Khiao area, Nakhon Ratchasima belonging to Loei Fold Belt during (A) Carboniferous to Early Permian, (B) Late Permian, and (C) Middle Triassic (modified from Fanka et al., 2018).

depths $(14-17 \mathrm{~km})$ and the calculated temperature range of crystallization $\left(861-927^{\circ} \mathrm{C}\right)$ indicate geothermal gradient of about $50-65^{\circ} \mathrm{C} / \mathrm{km}$ which is comparable with an active continental margin (Rothstein and Manning, 2003; Annen et al., 2006; Chi and Reed, 2008). The combination of crystallization temperatures, pressure, and depths indicates that their original magmas should have stored and partially crystallized in the middle continental crust, which are the same provenance reported for plutonic rocks (e.g., hornblende granite, biotitehornblende granite, biotite granite) in the adjacent area (Peacock, 1993; Winter, 2001; Kelemen et al., 2003; Richards, 2003; Fanka et al., 2018).

\section{Petrogenesis}

Based on petrochemistry, the studied volcanic rocks are classified as rhyolite, dacite, and andesite whereas the related dikes also show andesitic composition. Harker variation diagrams (Figure 6) present close relation of these rock types which reflect the same magmatic differentiation. Moreover, the most typical occurrences of hornblende in all rock types should imply crystallization of a hydrous magma (Foden and Green, 1992; Best,
2003). The hydrous magmatic model of these volcanic rocks and dikes is also supported by the hydrous calc-alkaline magmatism reported by Fanka et al. $(2016,2018)$ for the plutonic rocks in the Wang Nam Khiao area.

Enrichment of LILE (e.g., Rb, Sr) and depletion of HFSE (e.g., $\mathrm{Nb}, \mathrm{Ce}, \mathrm{Ti}$ ) presented in Figure 7A suggest an arc-derived magmatic affinity in a subduction zone (Pearce, 1983; Ryerson and Watson, 1987; Kelemen et al., 1990, 1993; Ringwood, 1990; Blein et al., 2001; Reubi and Nicholls, 2004; Jicha et al., 2004; Jicha and Singer, 2006; Fanka et al., 2016, 2018). In addition, REE patterns of these rock samples (Figure 7B) also reflect fractionated pattern of magmatism.

In summary, all characteristics of petrography, geochemistry, and mineral chemistry features of volcanic rocks and related dikes indicate clearly that these rocks are derived from the calcalkaline magmatic affinity related to continental arc margin. Their occurrences are supported by the multiple episodes of arc magmatism in the Wang Nam Khiao area, Nakhon Ratchasima suggested by Fanka et al. $(2016,2018)$ who have reported crucial evidences in terms of the ages of hornblendite and granitic rocks (Figure 1B). 


\section{Tectonic Implications}

According to the field evidence, the dike clearly intrudes into the Late Permian hornblende granite (Figure 3C); therefore, this dike probably formed during/after the emplacement of the Late Permian hornblende granite. Moreover, the formation of the studied volcanic rocks and dikes can be combined with other magmatism in the study area (Figure 1) reported as multiple arc magmatism comprising Carboniferous biotite granite, Late and Triassic biotite-hornblende granite, and Permian hornblendite and hornblende gabbro (Figure 8). Moreover, the magmatic events of the studied rocks can be comparable with the magmatism from both plutonic rocks (e.g., Zaw et al., 2007, 2014; Khositanont et al., 2008; Salam et al., 2014) and volcanic rocks (e.g., Qian et al., 2015) in other areas belonging to LFB (Figure 2).

Based on the geological data above together with the previous tectonic models in Thailand and SE Asia (e.g., Bunopas et al., 1989; Bunopas and Vella, 1992; Charusiri et al., 1993, 2002; Sone and Metcalfe, 2008; Barr and Charusiri, 2011; Searle et al., 2012; Zaw et al., 2014; Fanka et al., 2018), here, the tectonic evolution model indicating magmatism of the Wang Nam Khiao area in LFB is suggested in Figure 9.

The geological data have confirmed that the arc magmatisms along the LFB resulted from the Paleo-Tethys subduction beneath Indochina Terrane in several episodes (Fanka et al., 2016, 2018). According to geochronology of volcanic rocks in LFB, the magmatism might be started from Early Silurian (Khositanont et al., 2008) or Late Devonian (Intasopa and Dunn, 1994) as arc magmatism evidenced from the volcanic rocks in eastern volcanic sub-belt in Loei area. Moreover, these ages are supported by the ages of inherited zircons from Late Permian hornblende gabbro (Fanka et al., 2016) yielded zircon U-Pb ages of $444 \pm 10 \mathrm{Ma}$, and inherited zircons from Carboniferous biotite granite (Fanka et al., 2018) yielded zircon $\mathrm{U}-\mathrm{Pb}$ ages of $429 \pm 24 \mathrm{Ma}$ in the Wang Nam Khiao area (Figure 8).

In Carboniferous to Early Permian (Figure 9A), the subduction of Paleo-Tethys under Indochina Terrane (Bunopas, 1981; Sone and Metcalfe, 2008; Metcalfe, 2011, 2013; Fanka et al., 2018) generated the arc magmatism including Carboniferous biotite granite (zircon $\mathrm{U}-\mathrm{Pb}$ ages of 314.6-287.9 Ma) in Wang Nam Khiao area (Fanka et al., 2018) (Figure 8), which is consistent with the occurrence of the plutonic rocks in Phetchabun area (zircon $\mathrm{U}-\mathrm{Pb}$ ages of $323-310 \mathrm{Ma}$ from Zaw et al., 2007) (Figure 2), and volcanic rocks along LFB, e.g., from the Muang Feuang and Pak Lay regions, Laos (zircon U-Pb ages of 349.6-314.6 Ma from Qian et al., 2015) (Figure 2).

Afterward, during the Late Permian, the magmatisms strongly occurred in the LFB evidenced from the exposures of various arc magmatic rocks including volcanic rocks and dikes in this study, Late Permian hornblende granite (zircon $\mathrm{U}-\mathrm{Pb}$ ages of $253.4 \pm$ 4.3 Ma from Fanka et al., 2018), hornblendite, and hornblende gabbro (zircon $\mathrm{U}-\mathrm{Pb}$ ages of $257.1 \pm 3.4 \mathrm{Ma}$ from Fanka et al., 2016) in the Wang Nam Khiao area (Figures 2, 8). These rocks appear to have crystallized in different depths of the arc-related subduction between Paleo-Tethys and Indochina
Terrane (Figure 9B). Moreover, the magmatisms in this period have also been reported widely along LFB (Figure 2) (e.g., Khositanont et al., 2008; Salam et al., 2014; Zaw et al., 2014). They are composed of plutonic rocks (e.g., zircon U$\mathrm{Pb}$ ages of 254-250 Ma from Khositanont et al., 2008) and volcanic rocks (zircon $\mathrm{U}-\mathrm{Pb}$ ages of 258.6-245.9 Ma from Salam et al., 2014).

Subsequently, in Middle Triassic, the arc magmatism related to subduction of Palaeo-Tethys and Indochina Terrane (Figure 9C) had still proceeded along with the formation of Middle Triassic biotite-hornblende granite (zircon $\mathrm{U}-\mathrm{Pb}$ ages of $237.6 \pm$ 3.8 Ma from Fanka et al., 2018) (Figure 8) and continued colliding until the Late Triassic (Sone and Metcalfe, 2008; Ridd, 2012; Kamvong et al., 2014; Salam et al., 2014) or Middle Jurassic (Nualkhao et al., 2018). This magmatic event is supported by the occurrence of the plutonic rocks in Pak Chong area (zircon $\mathrm{U}-\mathrm{Pb}$ ages of $241 \pm 6 \mathrm{Ma}$ in Morley et al., 2013 and Arboit et al., 2016), Phetchabun area (zircon U-Pb ages of 249.2-245.9 Ma from Salam et al., 2014), Loei area (zircon U-Pb ages of $230 \pm 4 \mathrm{Ma}$, from Khositanont et al., 2008), and volcanic rocks in Loei area [zircon U-Pb ages of 245.9-241 Ma from Kamvong et al. (2014)] along LFB (Figure 9).

\section{CONCLUSION}

Volcanic rocks and related dikes in the Wang Nam Khiao area, Nakhon Ratchasima, Northeast Thailand, provide informative evidences of ancient magmatism and geological process as concluded in the following:

1. Volcanic rocks are petrochemically classified as rhyolite, dacite, and andesite whereas their related dikes yield andesitic composition. Plagioclase, hornblende, and quartz appear to be the main compositions in all rock types, but their proportions are obviously different.

2. Mineral composition, mineral chemistry, and geochemistry indicate the hydrous calc-alkaline magma resulted from continental arc subduction.

3. LILE (e.g., Rb, Sr) enrichment and HFSE depletion (e.g., $\mathrm{Nb}, \mathrm{Ce}, \mathrm{Ti})$ together with the slightly high LREE patterns indicate arc magmatism.

4. Al-in-hornblende geobarometer and hornblende thermometer indicate that the crystallization $\mathrm{P}-\mathrm{T}$ conditions of the phenocrysts, which ascend with the magma that subsequently fed the volcanic rocks and the andesitic dikes, range between 4.5 and $5.5 \mathrm{kbar}$ and $861-$ $972^{\circ} \mathrm{C}$, as well as $4.8-5.5 \mathrm{kbar}$ and $873-890^{\circ} \mathrm{C}$, respectively, reflecting the middle crust region.

5. Relationship of the dike cross-cutting into the previously dated Late Permian hornblende granite indicates that the volcanic rocks and dikes have taken place during/after Late Permian.

6. The results of this study well support the model of multiple arc magmatisms in LFB that resulted from the subduction of Palaeo-Tethys beneath Indochina Terrane. 


\section{DATA AVAILABILITY STATEMENT}

The raw data supporting the conclusions of this article will be made available by the authors, without undue reservation.

\section{AUTHOR CONTRIBUTIONS}

$\mathrm{VH}$ contributed to data collection, discussion, comment, and manuscript revision. CS contributed to data collection, comment, and manuscript revision. AF contributed to field investigation, laboratory studies, data collection, discussion, manuscript preparation, and revision. All authors contributed to the article and approved the submitted version.

\section{REFERENCES}

Anderson, J. L., and Smith, D. R. (1995). The effects of temperature and fO2 on the Al-in-hornblende barometer. Am. Mineral. 80, 549-559. doi: 10.2138/am1995-5-614

Annen, C., Blundy, J. D., and Sparks, R. S. J. (2006). The genesis of intermediate and silicic magmas in deep crustal hot zones. J. Petrol. 47, 505-539. doi: 10.1093/petrology/egi084

Arboit, F., Collins, A. S., Morley, C. K., Jourdan, F., King, R., Foden, J., et al. (2016). Geochronological and geochemical studies of mafic and intermediate dykes from the Khao Khwang Fold-thrust belt: implications for petrogenesis and tectonic evolution. Gondwana Res. 36, 124-141. doi: 10.1016/j.gr.2016. 04.005

Barr, S. M., and Charusiri, P. (2011). "Volcanic rocks in Thailand," in The Geology of Thailand, eds M. F. Ridd, A. J. Barber, and M. J. Crow (London: Geological Society), 415-439. doi: 10.1144/goth.15

Best, M. G. (2003). Igneous and Metamorphic Petrology. Oxford: Blackwell Science.

Bethel-Thompson, C., Sainsbury, J., Ricketts, J. W., and Girty, G. H. (2014). The rattlesnake valley and oriflamme canyon plutons: key temporal markers in the jurassic and cretaceous development of the transition zone of the peninsular ranges batholith. Geol. Soc. Am. 211, 609-623. doi: 10.1130/2014. 1211(18)

Blein, O., Lapierre, H., and Schweickert, R. A. (2001). A Permian island-arc with a continental basement: the black dyke formation nevada, North American Cordillera. Chem. Geol. 175, 543-566. doi: 10.1016/s0009-2541(00)00357-0

Boonsoong, A., Panjasawatwong, Y., and Metparsopsan, K. (2011). Petrochemistry and tectonic setting of mafic volcanic rocks in the chon daen-wang pong area, Phetchabun, Thailand. Isl. Arc. 20, 107-124. doi: 10.1111/j.1440-1738.2010. 00748.x

Bunopas, S. (1981). Palaeogeographic History of Western Thailand and Adjacent Parts of Southeast Asia: A Plate Tectonic Interpretation. Ph.D. thesis, Victoria University, Wellington.

Bunopas, S., Marante, S., and Vella, P. (1989). "Palaeozoic and early mesozoic rotation and drifting of shan-thai from Gondwana-Australia," in The Proceedings of the 4th International Symposium on Pre-Jurassic Evolution of East Asia, IGCP Project, 224, 63-64.

Bunopas, S., and Vella, P. (1992). "Geotectonic and geologic evolution of Thailand," in Proceedings of the National Conference on Geologic Resources of Thailand: Potential for Future Development, Department of Mineral Resource, Bangkok.

Charusiri, P., Clark, A. H., Farrar, E., Archibald, D., and Charusiri, B. (1993). Granite belts in Thailand: evidence from the 40Ar/ 39Ar geochronological and geological syntheses. J. Southeast Asian Earth Sci. 8, 127-136. doi: 10.1016/ 0743-9547(93)90014-g

Charusiri, P., Daorerk, V., Archibald, D., Hisada, K., and Am-paiwan, T. (2002). Geotectonic evolution of Thailand, a new synthesis. J. Geol. Soc. Thailand 1, $1-20$.

Chen, Z., Zen, Z., Wang, X., Zhang, Y., Yin, X., Chen, S., et al. (2017). Mineral chemistry indicates the petrogenesis of rhyolite from the Southwestern

\section{FUNDING}

The first author was supported by the 90th Anniversary of Chulalongkorn University Fund (Ratchadaphiseksomphot Endowment Fund), Graduate School, Chulalongkorn University for her M.Sc. study.

\section{ACKNOWLEDGMENTS}

The authors would like to acknowledge the Department of Geology, Faculty of Science, Chulalongkorn University for laboratory study.

Okinawa Trough. J. Ocean Univ. China 16, 1097-1108. doi: 10.1007/s11802017-3344-2

Chi, W., and Reed, D. L. (2008). Evolution of shallow, crustal thermal structure from subduction to collision: an example from Taiwan. Geol. Soc. Am. Bull. 120, 679-690. doi: 10.1130/b26210.1

Cobbing, E. J., Mallick, D. I. J., Pitfield, P. E. J., and Teoh, L. H. (1986). The granites of the Southeast Asian tin belt. J. Geol. Soc. Lond. 143, 537-550. doi: 10.1144/gsjgs.143.3.0537

Cobbing, E. J., Pitfield, P. E. J., Derbyshire, D. P. F., and Mallick, D. I. J. (1992). The Granites Of The Southeast Asian Tin Belt. Richmond: HMSO.

Costa, F., Andreastuti, S., Bouvet de Maisonneuve, C., and Pallister, J. S. (2013). Petrological insights into the storage conditions, and magmatic processes that yielded the centennial 2010 Merapi explosive eruption. J. Volcanol. Geotherm. Res. 261, 209-235. doi: 10.1016/j.jvolgeores.2012.12.025

Fanka, A. (2016). Petrogenesis of Plutonic Complex In Wang Nam Khiao Area Changwat Nakhon Ratchasima. Ph. D thesis, Chulalongkorn University, Thailand.

Fanka, A., Tsunogae, T., Daorerk, V., Tsutsumi, Y., Takamura, Y., Endo, T., et al. (2016). Petrochemistry and mineral chemistry of Late permian hornblendite and hornblende gabbro from the wang nam khiao area, nakhon ratchasima, Thailand: indication of palaeo-tethyan subduction. J. Asian Earth Sci. 130, 239-255. doi: 10.1016/j.jseaes.2016.11.018

Fanka, A., Tsunogae, T., Daorerk, V., Tsutsumi, Y., Takamura, Y., and Sutthirat, C. (2018). Petrochemistry and zircon U-Pb geochronology of granitic rocks in the wang nam khiao area, nakhon ratchasima, thailand: implications for petrogenesis and tectonic setting. J. Asian Earth Sci. 157, 92-118. doi: 10.1016/ j.jseaes.2017.08.025

Foden, J. D., and Green, D. H. (1992). Possible role of amphibole in the origin of andesite: some experimental and natural evidence. Contrib. Mineral. Petrol. 109, 479-493. doi: 10.1007/bf00306551

Hammarstrom, J. M., and Zen, E. (1986). Aluminum in hornblende; an empirical igneous geobarometer. Am. Mineral. 71, 1297-1313.

Harker, A. (1909). The Natural History Of Igneous Rocks. London: Methuen.

Helmy, H. M., Ahmed, A. F., El Mahallawi, M. M., and Ali, S. M. (2004). Pressure, temperature and oxygen fugacity conditions of calc-alkaline granitoids, Eastern Desert of Egypt, and tectonic implications. J. Afr. Earth. Sci. 38, 255-268. doi: 10.1016/j.jafrearsci.2004.01.002

Hollister, L. S., Grissom, G. C., Peters, E. K., Stowell, H. H., and Sisson, V. B. (1987). Confirmation of the empirical correlation of $\mathrm{Al}$ in hornblende with pressure of solidification of calc-alkaline plutons. Am. Mineral. 72, 231-239.

Hossain, I., Tsunogae, T., and Rajesh, H. M. (2009). Geothermobarometry and fluid inclusions of dioritic rocks in Bangladesh: implications for emplacement depth and exhumation rate. J. Asian Earth Sci. 34, 731-739. doi: 10.1016/j.jseaes.2008. 10.010

Hovelmann, J., Putnis, A., Geisler, T., Schmidt, B. C., and Golla-Schindler, U. (2010). The replacement of plagioclase feldspars by albite: observations from hydrothermal experiments. Contrib. Mineral. Petrol. 159, 43-59. doi: 10.1007/ s00410-009-0415-4 
Intasopa, S. (1993). Petrology and Geochronology Of The Volcanic Rocks Of The Central Thailand Volcanic Belt. Ph.D. thesis, University of New Brunswick, Fredericton.

Intasopa, S., and Dunn, T. (1994). Petrology and Sr-Nd isotopic systems of the basalts and rhyolites, Loei, Thailand. J. Asian Earth Sci. 9, 167-180. doi: 10. 1016/0743-9547(94)90073-6

Jicha, B. R., and Singer, B. S. (2006). Volcanic history and magmatic evolution of Seguam Island, Aleutian Island arc, Alaska. Geol. Soc. Am. Bull. 118, 805-822. doi: 10.1130/b25861.1

Jicha, B. R., Singer, B. S., Brophy, J. G., Fournelle, J. H., Johnson, C. M., Beard, B. L., et al. (2004). Variable impact of the subducted slab on aleutian island arc magma sources: evidence from $\mathrm{Sr}, \mathrm{Nd}, \mathrm{Pb}$, and $\mathrm{Hf}$ Isotopes and Trace Element Abundances. J. Petrol. 45, 1845-1875. doi: 10.1093/petrology/egh036

Johnson, M. C., and Rutherford, M. J. (1989). Experimental calibration of the aluminum-inhornblende geobarometer with application to Long Valley caldera (California) volcanic rocks. Geology 17, 837-841.

Jungyusuk, N., and Khositanont, S. (1992). "Volcanic rocks and associated mineralization in Thailand," in Proceedings of National Conference on Geologic Resources of Thailand: Potential for Future, Development, Amsterdam.

Kamvong, T., Zaw, K., Meffre, S., Maas, R., Stein, H., and Lai, C. K. (2014). Adakites in the truong son and loei fold belts, Thailand and Laos: genesis and implications for geodynamics and metallogeny. Gondwana Res. 26, 165-184. doi: 10.1016/j.gr.2013.06.011

Kelemen, P. B., Johnson, K. T. M., Kinzler, R. J., and Irving, A. J. (1990). High-fieldstrength element depletions in arc basalts due to mantle-magma interactions. Nature 345, 521-524. doi: 10.1038/345521a0

Kelemen, P. B., Rilling, J. L., Parmentier, E. M., Mehl, L., and Hacker, B. R. (2003). Thermal structure due to solid-state flow in the mantle wedge beneath arcs. Inside the subduction factory. AGU Monogr. 138, 293-311. doi: 10.1029/ 138GM13

Kelemen, P. B., Shimizu, N., and Dunn, T. (1993). Relative depletion of niobium in some arc magmas and the continental crust: partitioning of $\mathrm{K}, \mathrm{Nb}$, La and Ce during melt/rock reaction in the upper mantle. Earth Planet. Sci. Lett. 120, 111-134. doi: 10.1016/0012-821x(93)90234-z

Kemlheg, S., and Wichidchalermpong, A. (1992a). Geologic Map of Thailand 1:50000 Ban Sap Noi (5338III). Pretoria: Department of Mineral Resources.

Kemlheg, S., and Wichidchalermpong, A. (1992b). Geologic Map of Thailand 1:50000 Ban Sukhang (5338II). Pretoria: Department of Mineral Resources.

Khositanont, S., Panjasawatwong, Y., Ounchanum, P., Thanasuthipitak, T., Zaw, K., and Meffre, S. (2008). "Petrochemistry and zircon age determination of LoeiPhetchabun volcanic rocks," in Proceedings of the International Symposia on Geoscience Resources and Environments of Asian Terranes (GREAT 2008), 4th IGCP 516 and 5th APSEG, Bangkok.

Kromkhun, K., Baines, G., Satarugsa, P., and Foden, J. (2013). "Petrochemistry of volcanic and plutonic rocks in loei province, loei-petchabun fold belt, Thailand," in Proceedings of the 2nd International Conference on Geological and Environmental Sciences, Singapore.

Kuprubasi, N., Guctekin, A., Celebi, D., and Kirmaci, M. Z. (2014). Mineral chemical constraints on the petrogenesis of mafic and intermediate volcanic rocks from the Erciyes and Hasandag volcanoes, Central Turkey. Chem. Erde 74, 585-600. doi: 10.1016/j.chemer.2013.11.003

Lange, R. A., Frey, H. M., and Hector, J. (2009). A thermodynamic model for the plagioclase-liquid hygrometer/thermometer. Am. Mineral. 94, 494-506. doi: 10.2138/am.2009.3011

Leake, B. E., Woolley, A. R., Arps, C. E. S., Birch, W. D., Gilbert, M. C., Grice, J. D., et al. (1997). Nomenclature of amphiboles: report of the subcommittee on amphiboles of the international mineralogical association, commission on new minerals and mineral names. Am. Mineral. 82, 1019-1037.

Metcalfe, I. (2011). Palaeozoic-mesozoic history of SE Asia. J. Geol. Soc. Lond. 355, 7-35. doi: $10.1144 / \mathrm{sp} 355.2$

Metcalfe, I. (2013). Review Gondwana dispersion and Asian accretion: tectonic and paleogeographic evolution of eastern tethys. J. Asian Earth Sci. 66, 1-33. doi: 10.1016/j.jseaes.2012.12.020

Morley, C. K., Ampaiwan, P., Thanudamrong, S., Kuenphan, N., and Warren, J. (2013). Development of the khao khwang fold and thrust belt: implications for the geodynamic setting of Thailand and cambodia during the indosinian orogeny. J. Asian Earth Sci. 62, 705-719. doi: 10.1016/j.jseaes.2012.11.021
Nakapadungrat, S., and Putthapiban, P. (1992). "Granites and associated mineralization in Thailand," in Proceeding of National Conference On Geologic Resources of Thailand: Potential for Future Development, Department of Mineral Resources, Bangkok.

Ng, S. W. P., Chung, S. L., Robb, L. J., Searle, M. P., Ghani, A. A., Whitehouse, M. J., et al. (2015a). Petrogenesis of malaysian granitoids in the Southeast Asian tin belt: part 1. geochemical and Sr-Nd isotopic characteristics. Geol. Soc. Am. Bull. 127, 1209-1237. doi: 10.1130/B31213.1

Ng, S. W. P., Whitehouse, M. J., Searle, M. P., Robb, L. J., Ghani, A. A., Chung, S. L., et al. (2015b). Petrogenesis of Malaysian granitoids in the Southeast Asian Tin Belt: Part 2. U-Pb zircon geochronology and tectonic model. Geol. Soc. Am. Bull. 127, 1238-1258. doi: 10.1130/B31214.1

Nualkhao, P., Takahashi, R., Imai, A., and Charusiri, P. (2018). Petrochemistry of granitoids along the Loei Fold Belt, Northeastern Thailand. Resour. Geol. 68, 395-424. doi: 10.1111/rge.12176

Panjasawatwong, Y., Zaw, K., Chantaramee, S., Limtrakun, P., and Pirarai, K. (2006). Geochemistry and tectonic setting of the Central Loei volcanic rocks, Pak Chom area, Loei, northeastern Thailand. J. Asian Earth Sci. 26, 77-90. doi: 10.1016/j.jseaes.2004.09.008

Peacock, S. M. (1993). Large-scale hydration of the lithosphere above subducting slabs. Chem. Geol. 108, 49-59. doi: 10.1016/0009-2541(93)90317-c

Pearce, J. A. (1983). "Role of sub-continental lithosphere in magma genesis at active continental margins," in Continental Basalts And Mantle Xenoliths, eds C. J. Hawkesworth and M. J. Norry (Nantwich: Cardiff University), 230-249.

Putthaphiban, P., Vichidchalerpong, A., and Boonprasert, T. (1989a). Geologic Map of Thailand 1:50000 Amphoe Wang Nam Khiao (5337I). Pretoria: Department of Mineral Resources.

Putthaphiban, P., Vichidchalerpong, A., and Boonprasert, T. (1989b). Geologic Map of Thailand 1:50000 Ban Tha Wang Sai (5337IV). Pretoria: Department of Mineral Resources.

Qian, X., Feng, Q., Yang, W., Wang, Y., Chonglakmani, C., and Monjai, D. (2015). Arc-like volcanic rocks in NW Laos: geochronological and geochemical constraints and their tectonic implications. J. Asian Earth Sci. 98, 342-357. doi: 10.1016/j.jseaes.2014.11.035

Reubi, O., and Nicholls, I. A. (2004). Magmatic evolution at batur volcanic field, Bali, Indonesia: petrological evidence for polybaric fractional crystallization and implications for caldera-forming eruptions. J. Volcanol. Geotherm. Res. 138, 345-369. doi: 10.1016/j.jvolgeores.2004.07.009

Richards, J. P. (2003). Tectono-magmatic precursors for porphyry $\mathrm{Cu}-(\mathrm{Mo}-\mathrm{Au})$ deposit formation. Econ. Geol. 98, 1515-1533. doi: 10.2113/gsecongeo.98.8. 1515

Ridd, M. F. (2012). The role of strike-slip faults in the displacement of the Palaeotethys suture zone in Southeast Thailand. J. Asian Earth Sci. 51, 63-84. doi: 10.1016/j.jseaes.2012.01.018

Ridolfi, F., and Renzulli, A. (2012). Calcic amphiboles in calc-alkaline and alkaline magmas: thermobarometric and chemometric empirical equations valid up to $1,130^{\circ} \mathrm{C}$ and $2.2 \mathrm{GPa}$. Contrib. Mineral. Petrol. 163, 877-895. doi: 10.1007/ s00410-011-0704-6

Ridolfi, F., Renzulli, A., and Puerini, M. (2010). Stability and chemical equilibrium of amphibole in calc-alkaline magmas: an overview, new thermobarometric formulations and application to subduction-related volcanoes. Contrib. Mineral. Petrol. 160, 45-66. doi: 10.1007/s00410-009-0465-7

Ringwood, A. E. (1990). Slab-mantle interactions, 3: petrogenesis of intraplate magmas and structure of the upper mantle. Chem. Geol. 82, 187-207.

Rivera, M., Martin, H., Pennec, J. L., Thouret, J., Gourgaud, A., and Gerbe, M. (2017). Petro-geochemical constraints on the source and evolution of magmas at El Misti volcano (Peru). Lithos 26, 240-259. doi: 10.1016/j.lithos.2016.11.009

Rothstein, D. A., and Manning, C. E. (2003). Geothermal gradients in continental magmatic arcs: constraints from the eastern peninsular ranges batholith, Baja California, México. Geol. Soc. Am. 374, 337-354.

Ryerson, F. J., and Watson, E. B. (1987). Rutile saturation in magmas: implications for Ti-Nb-Ta depletion in orogenic rock series. Earth Planet. Sci. Lett. 86, 225-239. doi: 10.1016/0012-821x(87)90223-8

Salam, A., Zaw, K., Meffre, S., McPhie, J., and Lai, C. K. (2014). Geochemistry and geochronology of epithermal Au-hosted Chatree volcanic sequence: implication for tectonic setting of the Loei fold belt in central Thailand. Gondwana Res. 26, 198-217. doi: 10.1016/j.gr.2013.10.008 
Schmidt, M. W. (1992). Amphibole composition in tonalite as a function of pressure: an experimental calibration of the Al-in-hornblende barometer. Contrib. Mineral. Petrol. 110, 304-310. doi: 10.1007/bf00310745

Scott, J. A. J., Mather, T. A., Pyle, D. M., Rose, W. I., and Chigna, G. (2012). The magmatic plumbing system beneath santiaguito volcano, Guatemala. J. Volcanol. Geotherm. Res. 237-238, 54-68. doi: 10.1016/j.jvolgeores.2012.05. 014

Searle, M. P., Whitehouse, M. J., Robb, L. J., Ghani, A. A., Hutchison, C. S., Sone, M., et al. (2012). Tectonic evolution of the sibumasu-indochina terrane collision zone in Thailand and Malaysia: constrains from new U-Pb zircon chronology of SE Asian tin granitoids. J. Geol. Soc. 169, 489-500. doi: 10.1144/001676492011-107

Shapiro, L. (1975). Rapid analysis of silicate, carbonate, and phosphate rocksrevised edition. U.S. Geol. Surv. Bull. 1401:76.

Smith, J. V., and Brown, W. L. (1974). Feldspar Minerals. Cham: Springer.

Sone, M., and Metcalfe, I. (2008). Parallel Tethyan sutures in mainland Southeast Asia: new insights for Palaeo-Tethys closure and implications for the Indosinian orogeny. Tectonics 340, 166-179. doi: 10.1016/j.crte.2007.09.008

Sone, M., Metcalfe, I., and Chaodumrong, P. (2012). The chanthaburi terrane of southeastern thailand: stratigraphic confirmation as a disrupted segment of the sukhothai Arc. J. Asian Earth Sci. 61, 16-32. doi: 10.1016/j.jseaes.2012.08.021

Sun, S. S., and McDonough, W. F. (1989). "Chemical and isotopic systematics of oceanic basalts: implications for mantle composition and processes," in Magmatism in the Ocean Basins, eds A. D. Saunders and M. J. Norry (London: Geological Society), 313-345. doi: 10.1144/gsl.sp.1989.042. 01.19

Vivatpinyo, J., Charusiri, P., and Sutthirat, C. (2014). Volcanic rocks from q-prospect, chatree gold deposit, phichit province, north central thailand: indicators of ancient subduction. Arab. J. Sci. Eng. 39, 325-338. doi: 10.1007/ s13369-013-0839-z

Walker, B. Jr., Klemetti, E., Grunder, A., Dilles, J., Tepley, F., and Giles, D. (2013). Crystal reaming during the assembly, maturation, and waning of an eleven-million-year crustal magma cycle: thermobarometry of the aucanquilcha volcanic cluster. Contrib. Mineral. Petrol. 165, 663-682. doi: 10.1007/s00410012-0829-2
Waters, L. E., and Lange, R. A. (2015). An updated calibration of the plagioclaseliquid hygrometer-thermometer applicable to basalts through rhyolites. Am. Mineral. 100, 2172-2184. doi: 10.2138/am-2015-5232

Winchester, J. A., and Floyd, P. A. (1977). Geochemical discrimination of different magma series and their differentiation products using immobile elements. Chem. Geol. 20, 325-343. doi: 10.1016/0009-2541(77)90057-2

Winter, J. D. (2001). An Introduction To Igneous And Metamorphic Petrology. Upper Saddle River: Prentice-Hall.

Yuan, G., Cao, Y., Schulz, H., Hao, F., Gluyas, J., Liu, K., et al. (2019). A review of feldspar alteration and its geological significance in sedimentary basins: from shallow aquifers to deep hydrocarbon reservoirs. Earth Sci. Rev. 191, 114-140. doi: 10.1016/j.earscirev.2019.02.004

Zaw, K., Meffre, S., Lai, C. K., Santosh, M., Burrett, C., Graham, I. T., et al. (2014). Tectonics and metallogeny of mainland Southeast Asia-a review and contribution. Gondwana Res. 26, 5-30. doi: 10.1016/j.gr.2013.10.010

Zaw, K., Rodmanee, T., Khositanont, S., Thanasuthipitak, T., and Ruamkid, S. (2007). "Geology and genesis of Phu Thap Fah gold skarn deposit, northeastern Thailand: implications for reduced gold skarn formation and mineral exploration," in Proceedings of GEOTHAI'07 International Conference on Geology of Thailand, Bangkok.

Zhu, C., and Lu, P. (2009). Alkali feldspar dissolution and secondary mineral precipitation in batch systems: 3. Saturation states of product minerals and reaction paths. Geochim. Cosmochim. Acta 73, 3171-3200. doi: 10.1016/j.gca. 2009.03.015

Conflict of Interest: The authors declare that the research was conducted in the absence of any commercial or financial relationships that could be construed as a potential conflict of interest.

Copyright (c) 2020 Hunyek, Sutthirat and Fanka. This is an open-access article distributed under the terms of the Creative Commons Attribution License (CC BY). The use, distribution or reproduction in other forums is permitted, provided the original author(s) and the copyright owner(s) are credited and that the original publication in this journal is cited, in accordance with accepted academic practice. No use, distribution or reproduction is permitted which does not comply with these terms. 